\title{
Acceleration of Petaelectronvolt protons in the Galactic Centre
}

H.E.S.S. Collaboration: A. Abramowski ${ }^{1}$, F. Aharonian ${ }^{2,3,4}$, F. Ait Benkhali ${ }^{2}$, A.G. Akhperjanian ${ }^{5,4}$, E.O. Angüner ${ }^{6}$, M. Backes ${ }^{7}$, A. Balzer ${ }^{8}$, Y. Becherini ${ }^{9}$, J. Becker Tjus ${ }^{10}$, D. Berge ${ }^{11}$, S. Bernhard $^{12}$, K. Bernlöhr ${ }^{2}$, E. Birsin ${ }^{6}$, R. Blackwell ${ }^{13}$, M. Böttcher ${ }^{14}$, C. Boisson ${ }^{15}$, J. Bolmont ${ }^{16}$, P. Bordas ${ }^{2}$, J. Bregeon ${ }^{17}$, F. Brun ${ }^{18}$, P. Brun ${ }^{18}$, M. Bryan ${ }^{8}$, T. Bulik ${ }^{19}$, J. Carr ${ }^{20}$, S. Casanova ${ }^{21,2}$, N. Chakraborty ${ }^{2}$, R. Chalme-Calvet ${ }^{16}$, R.C.G. Chaves ${ }^{17,22}$, A, Chen $^{23}$, M. Chrétien ${ }^{16}$, S. Colafrancesco ${ }^{23}$, G. Cologna ${ }^{24}$, J. Conrad ${ }^{25,26}$, C. Couturier ${ }^{16}$, Y. Cui ${ }^{27}$, I.D. Davids ${ }^{14,7}$, B. Degrange ${ }^{28}$, C. Deil ${ }^{2}$, P. deWilt ${ }^{13}$, A. Djannati-Ataï ${ }^{29}$, W. Domainko ${ }^{2}$, A. Donath ${ }^{2}$, L.O'C. Drury ${ }^{3}$, G. Dubus ${ }^{30}$, K. Dutson ${ }^{31}$, J. Dyks ${ }^{32}$, M. Dyrda ${ }^{21}$, T. Edwards ${ }^{2}$, K. Egberts ${ }^{33}$, P. Eger ${ }^{2}$, J.-P. Ernenwein ${ }^{20}$, P. Espigat ${ }^{29}$, C. Farnier $^{25}$, S. Fegan ${ }^{28}$, F. Feinstein ${ }^{17}$, M.V. Fernandes ${ }^{1}$, D. Fernandez ${ }^{17}$, A. Fiasson ${ }^{34}$, G. Fontaine ${ }^{28}$, A. Förster ${ }^{2}$, M. Füßling ${ }^{35}$, S. Gabici ${ }^{29}$, M. Gajdus ${ }^{6}$, Y.A. Gallant ${ }^{17}$, T. Garrigoux ${ }^{16}$, G. Giavitto $^{35}$, B. Giebels ${ }^{28}$, J.F. Glicenstein ${ }^{18}$, D. Gottschall ${ }^{27}$, A. Goyal ${ }^{36}$, M.-H. Grondin ${ }^{37}$, M. Grudzińska ${ }^{19}$, D. Hadasch ${ }^{12}$, S. Häffner ${ }^{38}$, J. Hahn ${ }^{2}$, J. Hawkes ${ }^{13}$, G. Heinzelmann ${ }^{1}$, G. Henri ${ }^{30}$, G. Hermann ${ }^{2}$, O. Hervet ${ }^{15}$, A. Hillert ${ }^{2}$, J.A. Hinton ${ }^{2,31}$, W. Hofmann ${ }^{2}$, P. Hofverberg ${ }^{2}$, C. Hoischen ${ }^{33}$, M. Holler ${ }^{28}$, D. Horns ${ }^{1}$, A. Ivascenko ${ }^{14}$, A. Jacholkowska ${ }^{16}$, M. Jamrozy ${ }^{36}$, M. Janiak ${ }^{32}$, F. Jankowsky ${ }^{24}$, I. Jung-Richardt ${ }^{38}$, M.A. Kastendieck ${ }^{1}$, K. Katarzyński ${ }^{39}$, U. Katz ${ }^{38}$, D. Kerszberg ${ }^{16}$, B. Khélifi ${ }^{29}$, M. Kieffer ${ }^{16}$, S. Klepser ${ }^{35}$, D. Klochkov ${ }^{27}$, W. Kluźniak ${ }^{32}$, D. Kolitzus ${ }^{12}$, Nu. Komin ${ }^{23}$, K. Kosack ${ }^{18}$, S. Krakau ${ }^{10}$, F. Krayzel ${ }^{34}$, P.P. Krüger ${ }^{14}$, H. Laffon ${ }^{37}$, G. Lamanna ${ }^{34}$, J. Lau ${ }^{13}$, J. Lefaucheur ${ }^{29}$, V. Lefranc ${ }^{18}$, A. Lemière ${ }^{29}$, M. Lemoine-Goumard ${ }^{37}$, J.-P. Lenain ${ }^{16}$, T. Lohse ${ }^{6}$, A. Lopatin ${ }^{38}$, C.-C. Lu² , R. Lui ${ }^{2}$, V. Marandon ${ }^{2}$, A. Marcowith ${ }^{17}$, C. Mariaud ${ }^{28}$, R. Marx ${ }^{2}$, G. Maurin ${ }^{34}$, N. Maxted ${ }^{17}$, M. Mayer ${ }^{6}$, P.J. Meintjes ${ }^{40}$, U. Menzler ${ }^{10}$, M. Meyer ${ }^{25}$, A.M.W. Mitchell ${ }^{2}$, R. Moderski ${ }^{32}$, M. Mohamed ${ }^{24}$, K. Morå25 ${ }^{25}$ E. Moulin ${ }^{18}$, T. Murach $^{6}$, M. de Naurois ${ }^{28}$, J. Niemiec ${ }^{21}$, L. Oakes ${ }^{6}$, H. Odaka ${ }^{2}$, S. Öttl ${ }^{12}$, S. Ohm ${ }^{35}$, B. Opitz ${ }^{1}$, M. Ostrowski ${ }^{36}$, I. Oya ${ }^{35}$, M. Panter ${ }^{2}$, R.D. Parsons ${ }^{2}$, M. Paz Arribas ${ }^{6}$, N.W. Pekeur ${ }^{14}$, G. Pelletier ${ }^{30}$, P.-O. Petrucci ${ }^{30}$, B. Peyaud ${ }^{18}$, S. Pita ${ }^{29}$, H. Poon ${ }^{2}$, H. Prokoph ${ }^{9}$, G. Pühlhofer ${ }^{27}$, M. Punch ${ }^{29}$, A. Quirrenbach ${ }^{24}, S_{\text {S. } \text { Raab }^{38}, \text { I. Reichardt }}{ }^{29}$, A. Reimer ${ }^{12}$, O. Reimer ${ }^{12}$, M. Renaud ${ }^{17}$, R. de los Reyes ${ }^{2}$, F. Rieger ${ }^{2,41}$, C. Romoli ${ }^{3}$, S. Rosier-Lees ${ }^{34}$, G. Rowell ${ }^{13}$, B. Rudak ${ }^{32}$, C.B. Rulten ${ }^{15}$, V. Sahakian ${ }^{5,4}$, D. Salek ${ }^{42}$, D.A. Sanchez ${ }^{34}$, A. Santangelo ${ }^{27}$, M. Sasaki ${ }^{27}$, R. Schlickeiser ${ }^{10}$, F. Schüssler ${ }^{18}$, A. Schulz ${ }^{35}$, U. Schwanke ${ }^{6}$, S. Schwemmer ${ }^{24}$, A.S. Seyffert ${ }^{14}$, R. Simoni ${ }^{8}$, H. Sol ${ }^{15}$, F. Spanier ${ }^{14}$, G. Spengler ${ }^{25}$, F. Spies ${ }^{1}$, Ł. Stawarz ${ }^{36}$, R. Steenkamp ${ }^{7}$, C. Stegmann ${ }^{33,35}$, F. Stinzing ${ }^{38}$, K. Stycz ${ }^{35}$, I. Sushch ${ }^{14}$, J.-P. Tavernet ${ }^{16}$, T. Tavernier ${ }^{29}$, A.M. Taylor ${ }^{3}$, R. Terrier ${ }^{29}$, M. Tluczykont ${ }^{1}$, C. Trichard $^{34}$, R. Tuffs ${ }^{2}$, K. Valerius ${ }^{38}$, J. van der Walt ${ }^{14}$, C. van Eldik ${ }^{38}$, B. van Soelen ${ }^{40}$, G. Vasileiadis ${ }^{17}$, J. Veh ${ }^{38}$, C. Venter ${ }^{14}$, A. Viana ${ }^{2}$, P. Vincent ${ }^{16}$, J. Vink ${ }^{8}$, F. Voisin ${ }^{13}$, H.J. Völk ${ }^{2}$, T. Vuillaume ${ }^{30}$, S.J. Wagner ${ }^{24}$, P. Wagner ${ }^{6}$, R.M. Wagner ${ }^{25}$, M. Weidinger ${ }^{10}$, Q. Weitzel ${ }^{2}$, R. White ${ }^{31}$, A. Wierzcholska ${ }^{24,21}$, P. Willmann ${ }^{38}$, A. Wörnlein ${ }^{38}$, D. Wouters ${ }^{18}$, R. Yang ${ }^{2}$, V. Zabalza ${ }^{31}$, D. Zaborov ${ }^{28}$, M. Zacharias ${ }^{24}$, A.A. Zdziarski ${ }^{32}$, A. Zech ${ }^{15}$, F. Zefi ${ }^{28}$, N. Żywucka ${ }^{36}$

${ }^{1}$ Universität Hamburg, Institut für Experimentalphysik, Luruper Chaussee 149, D 22761 Hamburg, Germany

${ }^{2}$ Max-Planck-Institut für Kernphysik, P.O. Box 103980, D 69029 Heidelberg, Germany

${ }^{3}$ Dublin Institute for Advanced Studies, 31 Fitzwilliam Place, Dublin 2, Ireland 
${ }^{4}$ National Academy of Sciences of the Republic of Armenia, Marshall Baghramian Avenue, 24, 0019 Yerevan, Republic of Armenia

${ }^{5}$ Yerevan Physics Institute, 2 Alikhanian Brothers St., 375036 Yerevan, Armenia

${ }^{6}$ Institut für Physik, Humboldt-Universität zu Berlin, Newtonstr. 15, D 12489 Berlin, Germany

${ }^{7}$ University of Namibia, Department of Physics, Private Bag 13301, Windhoek, Namibia

${ }^{8}$ GRAPPA, Anton Pannekoek Institute for Astronomy, University of Amsterdam, Science Park 904, 1098 XH Amsterdam, The Netherlands

${ }^{9}$ Department of Physics and Electrical Engineering, Linnaeus University, 35195 Växjö, Sweden

${ }^{10}$ Institut für Theoretische Physik, Lehrstuhl IV: Weltraum und Astrophysik, Ruhr-Universität Bochum, D 44780 Bochum, Germany

${ }^{11}$ GRAPPA, Anton Pannekoek Institute for Astronomy and Institute of High-Energy Physics, University of Amsterdam, Science Park 904, 1098 XH Amsterdam, The Netherlands

${ }^{12}$ Institut für Astro- und Teilchenphysik, Leopold-Franzens-Universität Innsbruck, A-6020 Innsbruck, Austria

${ }^{13}$ School of Chemistry \& Physics, University of Adelaide, Adelaide 5005, Australia

${ }^{14}$ Centre for Space Research, North-West University, Potchefstroom 2520, South Africa

${ }^{15}$ LUTH, Observatoire de Paris, CNRS, Université Paris Diderot, 5 Place Jules Janssen, 92190 Meudon, France

${ }^{16}$ LPNHE, Université Pierre et Marie Curie Paris 6, Université Denis Diderot Paris 7, CNRS/IN2P3, 4 Place Jussieu, F-75252, Paris Cedex 5, France

${ }^{17}$ Laboratoire Univers et Particules de Montpellier, Université Montpellier 2, CNRS/IN2P3, CC 72, Place Eugène Bataillon, F-34095 Montpellier Cedex 5, France

${ }^{18}$ DSM/Irfu, CEA Saclay, F-91191 Gif-Sur-Yvette Cedex, France

${ }^{19}$ Astronomical Observatory, The University of Warsaw, Al. Ujazdowskie 4, 00-478 Warsaw, Poland

${ }^{20}$ Aix Marseille Universié, CNRS/IN2P3, CPPM UMR 7346, 13288 Marseille, France

${ }^{21}$ Instytut Fizyki Jạdrowej PAN, ul. Radzikowskiego 152, 31-342 Kraków, Poland

${ }^{22}$ Funded by EU FP7 Marie Curie, grant agreement No. PIEF-GA-2012-332350,

${ }^{23}$ School of Physics, University of the Witwatersrand, 1 Jan Smuts Avenue, Braamfontein, Johannesburg, 2050 South Africa

${ }^{24}$ Landessternwarte, Universität Heidelberg, Königstuhl, D 69117 Heidelberg, Germany

${ }^{25}$ Oskar Klein Centre, Department of Physics, Stockholm University, Albanova University Center, SE10691 Stockholm, Sweden

${ }^{26}$ Wallenberg Academy Fellow,

${ }^{27}$ Institut für Astronomie und Astrophysik, Universität Tübingen, Sand 1, D 72076 Tübingen, Germany

${ }^{28}$ Laboratoire Leprince-Ringuet, Ecole Polytechnique, CNRS/IN2P3, F-91128 Palaiseau, France

${ }^{29}$ APC, AstroParticule et Cosmologie, Université Paris Diderot, CNRS/IN2P3, CEA/Irfu, Observatoire de Paris, Sorbonne Paris Cité, 10, rue Alice Domon et Léonie Duquet, 75205 Paris Cedex 13, France

${ }^{30}$ Univ. Grenoble Alpes, IPAG, F-38000 Grenoble, France

CNRS, IPAG, F-38000 Grenoble, France

${ }^{31}$ Department of Physics and Astronomy, The University of Leicester, University Road, Leicester, LE1 7RH, United Kingdom

${ }^{32}$ Nicolaus Copernicus Astronomical Center, ul. Bartycka 18, 00-716 Warsaw, Poland

${ }^{33}$ Institut für Physik und Astronomie, Universität Potsdam, Karl-Liebknecht-Strasse 24/25, D 14476 Potsdam, Germany

${ }^{34}$ Laboratoire d'Annecy-le-Vieux de Physique des Particules, Université Savoie Mont-Blanc, CNRS/IN2P3, 
F-74941 Annecy-le-Vieux, France

${ }^{35}$ DESY, D-15738 Zeuthen, Germany

${ }^{36}$ Obserwatorium Astronomiczne, Uniwersytet Jagielloński, ul. Orla 171, 30-244 Kraków, Poland

${ }^{37}$ Université Bordeaux, CNRS/IN2P3, Centre d'Études Nucléaires de Bordeaux Gradignan, 33175 Gradignan, France

${ }^{38}$ Universität Erlangen-Nürnberg, Physikalisches Institut, Erwin-Rommel-Str. 1, D 91058 Erlangen, Germany

${ }^{39}$ Centre for Astronomy, Faculty of Physics, Astronomy and Informatics, Nicolaus Copernicus University, Grudziadzka 5, 87-100 Torun, Poland

${ }^{40}$ Department of Physics, University of the Free State, PO Box 339, Bloemfontein 9300, South Africa

${ }^{41}$ Heisenberg Fellow (DFG), ITA Universität Heidelberg, Germany,

${ }^{42}$ GRAPPA, Institute of High-Energy Physics, University of Amsterdam, Science Park 904, 1098 XH Amsterdam, The Netherlands

Galactic cosmic rays reach energies of at least a few Peta-electronvolts $\left(\mathbf{P e V}=10^{15}\right.$ electron volts) ${ }^{1}$. This implies our Galaxy contains PeV accelerators (PeVatrons), but all proposed models of Galactic cosmic-ray accelerators encounter non-trivial difficulties at exactly these energies $^{2}$. Tens of Galactic accelerators capable of accelerating particle to tens of TeV (1 TeV $=10^{12}$ electron volts) energies were inferred from recent gamma-ray observations ${ }^{3}$. None of the currently known accelerators, however, not even the handful of shell-type supernova remnants commonly believed to supply most Galactic cosmic rays, have shown the characteristic tracers of $\mathrm{PeV}$ particles: power-law spectra of gamma rays extending without a cutoff or a spectral break to tens of $\mathrm{TeV}^{\sqrt{4}}$. Here we report deep gamma-ray observations with arcminute angular resolution of the Galactic Centre regions, which show the expected tracer of the presence of $\mathrm{PeV}$ particles within the central 10 parsec of the Galaxy. We argue that the supermassive black hole Sagittarius A* is linked to this PeVatron. Sagittarius A* went through active phases in the past, as demonstrated by $X$-ray outburst ${ }^{5}$ and an outflow from the Galactic Center ${ }^{6}$. Although its current rate of particle acceleration is not sufficient to provide a substantial contribution to Galactic cosmic rays, Sagittarius $A^{*}$ could have plausibly been more active over the last $\gtrsim 10^{6-7}$ years, and therefore should be considered as a viable alternative to supernova remnants as a source of PeV Galactic cosmic rays.

The large photon statistics accumulated over the last 10 years of observations with the High Energy Stereoscopic System (H.E.S.S.), together with improvements in the methods of data analysis, allow for a deep study of the properties of the diffuse very-high-energy (VHE; more than 100 $\mathrm{GeV}$ ) emission of the central molecular zone. This region surrounding the Galactic Centre contains predominantly molecular gas and extends (in projection) out to $\mathrm{r} \sim 250 \mathrm{pc}$ at positive galactic longitudes and $\mathrm{r} \sim 150 \mathrm{pc}$ at negative longitudes. The map of the central molecular zone as seen in VHE $\gamma$-rays (Fig. 1) shows a strong (although not linear; see below) correlation between the brightness distribution of VHE $\gamma$-rays and the locations of massive gas-rich complexes. This points towards a hadronic origin of the diffuse emission ${ }^{7}$, where the $\gamma$-rays result from the interactions of relativistic protons with the ambient gas. The second important mechanism of production of VHE $\gamma$-rays 
is the inverse Compton scattering of electrons. However, the severe radiative losses suffered by multi-TeV electrons in the Galactic Centre region prevent them from propagating over scales comparable to the size of the central molecular zone, thus disfavouring a leptonic origin of the $\gamma$-rays (see discussion in Methods and Extended Data Figures 1 and 2).

The location and the particle injection rate history of the cosmic-ray accelerator(s), responsible for the relativistic protons, determine the spatial distribution of these cosmic rays which, together with the gas distribution, shape the morphology of the central molecular zone seen in VHE $\gamma$-rays. Fig. 2 shows the radial profile of the $E \geq 10 \mathrm{TeV}$ cosmic-rays energy density $w_{\mathrm{CR}}$ up to $r \sim 200 \mathrm{pc}$ (for a Galactic Centre distance of $8.5 \mathrm{kpc}$ ), determined from the $\gamma$-ray luminosity and the amount of target gas (see Extended Data Tables 1 and 2). This high energy density in the central molecular zone is found to be an order of magnitude larger than that of the "sea" of cosmic rays that universally fills the Galaxy, while the energy density of low energy $(\mathrm{GeV})$ cosmic rays in this region has a level comparable to $\mathrm{it}^{\frac{8}{6}}$. This requires the presence of one or more accelerators of multi-TeV particles operating in the central molecular zone.

If the accelerator injects particles at a continuous rate, $\dot{Q}_{p}(E)$, the radial distribution of cosmic rays in the central molecular zone, in the case of diffusive propagation, is described as $w_{\mathrm{CR}}(E, r, t)=\frac{\dot{Q}_{p}(E)}{4 \pi D(E) r} \operatorname{erfc}\left(r / r_{\text {diff }}\right)^{9}$, where $D(E)$ and $r_{\text {diff }}$ are the diffusion coefficient and radius, respectively. For timescales smaller than the proton-proton interaction time in the hydrogen gas of density $n, t \leq t_{\mathrm{pp}} \simeq 5 \times 10^{4}\left(n / 10^{3} \mathrm{~cm}^{-3}\right)^{-1} \mathrm{yr}$, the diffusion radius is $r_{\text {diff }} \approx \sqrt{4 D(E) t}$. Thus, at distances $r<r_{\text {diff }}$, the proton flux should decrease as $\sim 1 / r$ provided that the diffusion coefficient does not significantly vary throughout the central molecular zone. The measurements clearly support the $w_{\mathrm{CR}}(r) \propto 1 / r$ dependence over the entire central molecular zone region (Fig. 2) and disfavour a $w_{\mathrm{CR}}(r) \propto 1 / r^{2}$ and a $w_{\mathrm{CR}}(r) \propto$ constant profiles, as expected if cosmic rays are advected in a wind, and in the case of a single burst-like event of cosmic-ray injection, respectively. The $1 / r$ profile of the cosmic-ray density up to $200 \mathrm{pc}$ indicates a quasi-continuous injection of protons into the central molecular zone from a centrally located accelerator on a timescale $\Delta t$ exceeding the characteristic time of diffusive escape of particles from the central molecular zone, i.e. $\Delta t \geq t_{\text {diff }} \approx R^{2} / 6 D \approx 2 \times 10^{3}\left(D / 10^{30} \mathrm{~cm}^{2} \mathrm{~s}^{-1}\right)^{-1} \mathrm{yr}$, where $D$ is normalised to the characteristic value of multi-TeV cosmic rays in the Galactic Disk ${ }^{10}$. In this regime the average injection rate of particles is found to be $\dot{Q}_{p}(\geq 10 \mathrm{TeV}) \approx 4 \times 10^{37}\left(\mathrm{D} / 10^{30} \mathrm{~cm}^{2} \mathrm{~s}^{-1}\right) \mathrm{erg} / \mathrm{s}$. The diffusion coefficient itself depends on the power spectrum of the turbulent magnetic field, which is highly unknown in the central molecular zone region. This introduces an uncertainty in the estimates of the injection power of relativistic protons. Yet, the diffusive nature of the propagation is constrained by the condition $R^{2} / 6 D \gg R / c$. For the radius of the central molecular zone region of $200 \mathrm{pc}$, this implies $D \ll 3 \times 10^{30} \mathrm{~cm}^{2} / \mathrm{s}$, and, consequently, $\dot{Q}_{p} \ll 1.2 \times 10^{38} \mathrm{erg} / \mathrm{s}$.

The energy spectrum of the diffuse $\gamma$-ray emission (Fig. 3) has been extracted from an annulus centred at Sagittarius (Sgr) A* (see Fig. 1). The best-fit to the data is found for a spectrum following a power law extending with a photon index $\approx 2.3$ to energies up to tens of $\mathrm{TeV}$, without any indication of a cutoff or a break. This is the first time that such a $\gamma$-ray spectrum, arising from hadronic interactions, is detected in general. Since these $\gamma$-rays result from the decay of neutral 
pions produced by $p p$ interactions, the derivation of such hard power-law spectrum implies that the spectrum of the parent protons should extend to energies close to $1 \mathrm{PeV}$. The best fit of a $\gamma$-ray spectrum from neutral pion decay to the H.E.S.S. data is found for a proton spectrum following a pure power-law with index $\approx 2.4$. We note that $p p$ interactions of $1 \mathrm{PeV}$ protons could also be studied by the observation of emitted neutrinos or the X-rays from the synchrotron emission of secondary electrons and positrons (see Methods and Extended Data Figures 3 and 4). However, the measured $\gamma$-ray flux puts the expected fluxes of neutrinos and X-rays below or at best close to the sensitivities of the current instruments. Assuming a cutoff in the parent proton spectrum, the corresponding secondary $\gamma$-ray spectrum deviates from the H.E.S.S. data at $68 \%, 90 \%$ and $95 \%$ confidence levels for cutoffs at 2.9 PeV, 0.6 PeV and 0.4 PeV, respectively. This is the first robust detection of a VHE cosmic hadronic accelerator which operates as a PeVatron.

Remarkably, the Galactic Centre PeVatron appears to be located in the same region as the

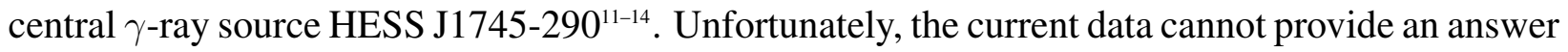
as to whether there is an intrinsic link between these two objects. The point-like source HESS J1745-290 itself remains unidentified. Besides Sgr A*15, other potential counterparts are the pulsar wind nebula $\mathrm{G}$ 359.95-0.04 ${ }^{16 / 17}$, and a spike of annihilating dark matter ${ }^{\sqrt{18}}$. Moreover, it has also been suggested that this source might have a diffuse origin, peaking towards the direction of the Galactic Centreg because of the higher concentration of both gas and relativistic particles ${ }^{15}$. In fact, this interpretation would imply an extension of the spectrum of the central source to energies beyond $10 \mathrm{TeV}$, which however is at odds with the detection of a clear cutoff in the spectrum of HESS J1745-290 at about $10 \mathrm{TeV} \sqrt{1920}$ (Fig. 3). Yet, the attractive idea of explaining the entire $\gamma$-ray emission from the Galactic Centre by run-away protons from the same centrally located accelerator can be still compatible with the cutoff in the spectrum of the central source. For example, the cutoff could be due to the absorption of $\gamma$-rays caused by interactions with the ambient infrared radiation field. It should be noted that although the question on the link between the central $\gamma$-ray source and the proton PeVatron is an interesting issue in its own right, it, however, does not have a direct impact on the main conclusions of this work.

The integration of the cosmic-ray radial distribution (Fig. 2) yields the total energy of $\mathrm{E} \geq 10$ $\mathrm{TeV}$ protons confined in the central molecular zone: $W_{\mathrm{CR}} \approx 1.0 \times 10^{49} \mathrm{erg}$. A single Supernova Remnant (SNR) would suffice to provide this rather modest energy in cosmic rays. A possible candidate could be Sgr A East. Although this object has already been excluded as a counterpart of HESS J1745-29021, the multi-TeV protons accelerated by this object and then injected into the central molecular zone could contribute to the diffuse $\gamma$-ray component. Another potential site for acceleration of protons in the Galactic Centre are the compact stellar clusters ${ }^{22}$. Formally, the mechanical power in these clusters in the form of stellar winds, which can provide adequate conditions for particle acceleration, is sufficient to explain the required total energy of cosmic rays in the central molecular zone. However, the acceleration of protons to $\mathrm{PeV}$ energies requires bulk motions in excess of $10,000 \mathrm{~km} / \mathrm{s}$ which in the stellar clusters could only exist because of very young supernova shocks ${ }^{23}$. Thus, the operation of PeVatrons in stellar clusters is reduced to the presence of supernovae shocks. On the other hand, since the acceleration of PeV particles by shocks, either in the individual SNRs or in the stellar clusters, cannot last significantly longer than 
100 years ${ }^{24}$, we would need more than 10 supernova events to meet the requirement of continuous injection of cosmic rays in the central molecular zone over $\gg 10^{3}$ years. For the central $10 \mathrm{pc}$ region, such a high supernova rate seems unlikely.

We suggest that the supermassive black hole at the Galactic Centre ( $\operatorname{Sgr} \mathrm{A}^{*}$ ) is the most plausible supplier of ultra-relativistic protons and nuclei accelerated either in the accretion flow, i.e. in the immediate vicinity of the black hole ${ }^{15 / 25}$ or somewhat further away, e.g. at the site of termination of an outflow ${ }^{26}$. If Sgr A* is indeed the particles' source, the required acceleration rate of about $10^{37-38} \mathrm{erg} / \mathrm{s}$ would exceed by two or three orders of magnitude the current bolometric luminosity of $\operatorname{Sgr} \mathrm{A}^{* 27}$, and would constitute at least $1 \%$ of the current accretion power of the supermassive black hole. Given the fact that the accretion rate in the central black hole currently is relatively modest, and that at certain epochs the black hole of $4 \times 10^{6}$ solar masses can operate at much higher accretion rate, we may speculate that this could facilitate also higher cosmic-ray production rates ${ }^{25}$. In this regard it is interesting to note that an average acceleration rate of $10^{39}$ $\mathrm{erg} / \mathrm{s}$ of $\mathrm{E}>10 \mathrm{TeV}$ protons over the last $10^{6-7}$ years would be sufficient to explain the flux of cosmic rays around the so-called "knee" at $1 \mathrm{PeV}$. If so, this could be a solution to one of the most controversial and actively debated problems of the paradigm of the SNR origin of Galactic cosmic rays ${ }^{24,2829}$. 

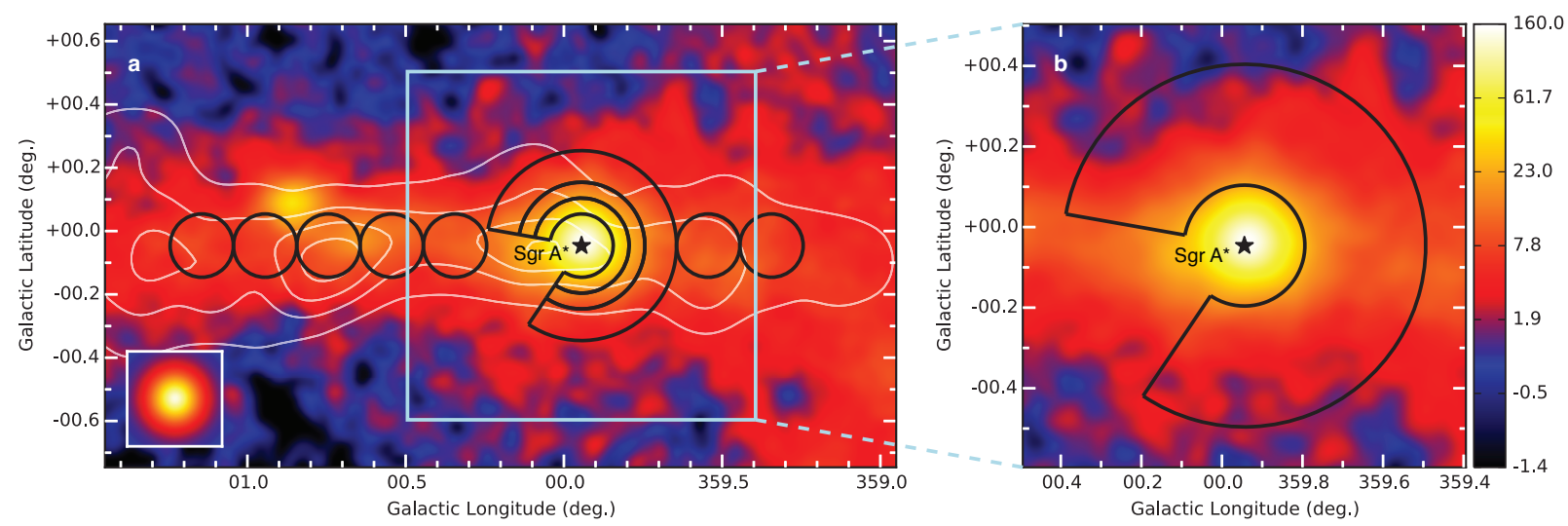

Figure 1: VHE $\gamma$-ray image of the Galactic Centre region. The colour scale indicates counts per $0.02^{\circ} \times 0.02^{\circ}$ pixel. Left panel: The black lines outline the regions used to calculate the CR energy density throughout the central molecular zone. A section of $66^{\circ}$ is excluded from the annuli (see Methods). White contour lines indicate the density distribution of molecular gas, as traced by its CS line emission ${ }^{30}$. The inset shows the simulation of a point-like source. Right panel: Zoomed view of the inner $\sim 70 \mathrm{pc}$ and the contour of the region used to extract the spectrum of the diffuse emission. 


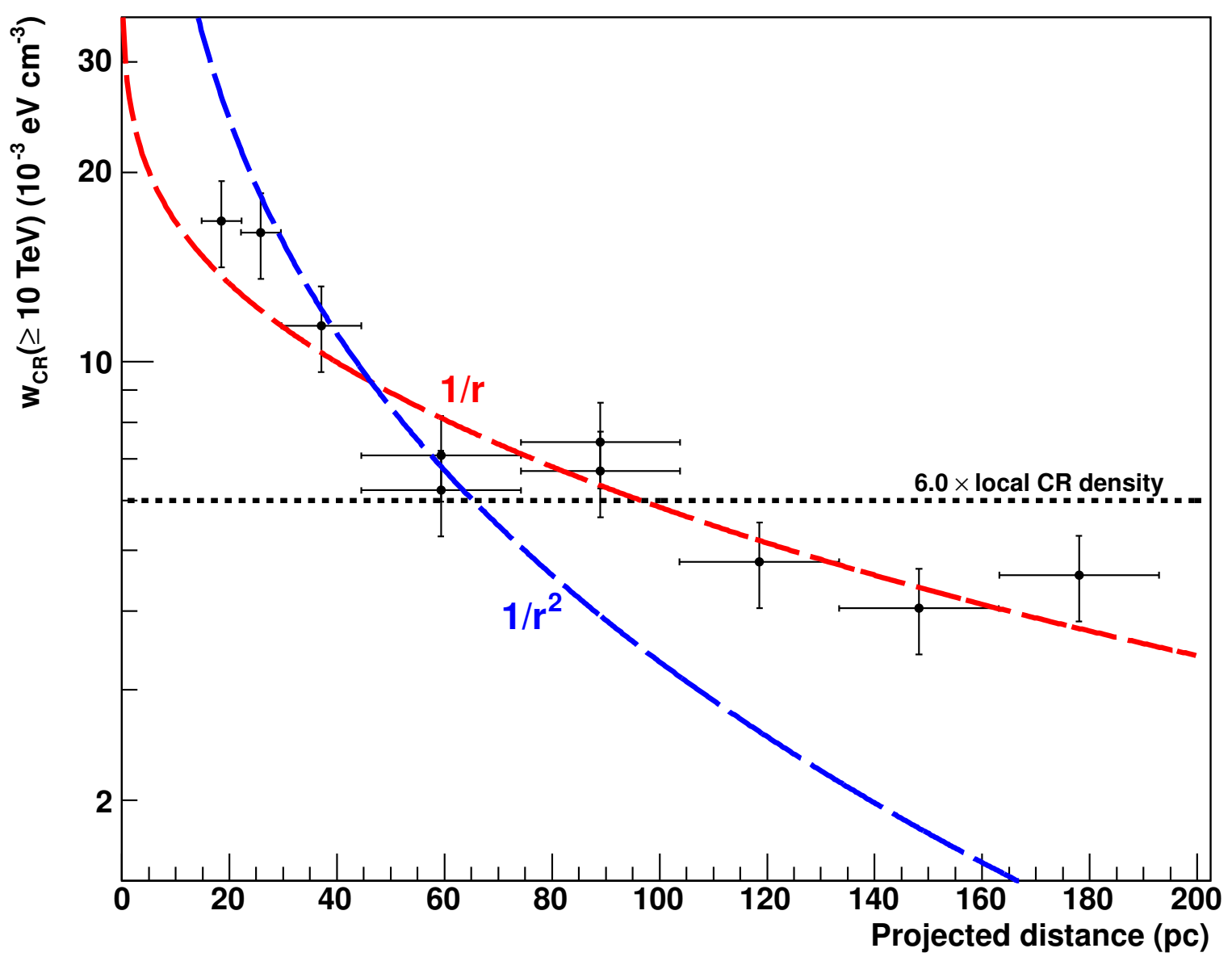

Figure 2: Spatial distribution of the CR density versus projected distance from $\operatorname{Sgr} \mathbf{A} *$. The vertical and horizontal error bars show the $1 \sigma$ statistical plus systematical errors and the bin size, respectively. A fit to the data of a $1 / r$ (red line, $\chi^{2} /$ d.o.f. $\left.=11.8 / 9\right), 1 / r^{2}$ (blue line, $\chi^{2} /$ d.o.f. $\left.=73.2 / 9\right)$ and an homogeneous (black line, $\chi^{2} /$ d.o.f. $\left.=61.2 / 9\right)$ CR density radial profiles integrated along the line of sight are shown. The best fit of a $1 / r^{\alpha}$ profile to the data is found for $\alpha=1.10 \pm 0.12(1 \sigma)$. The $1 / r$ radial profile is clearly preferred by the H.E.S.S. data. 


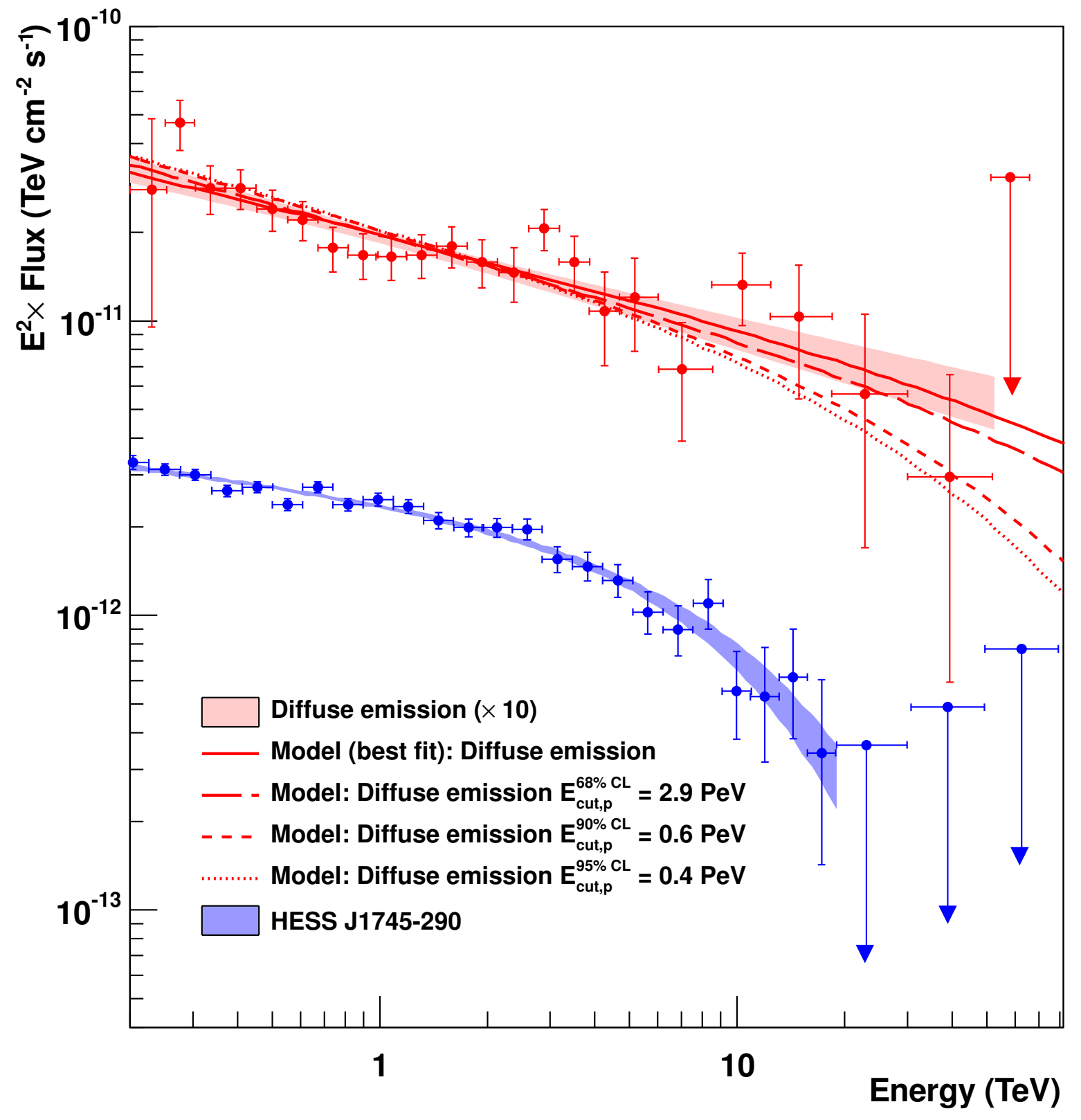

Figure 3: VHE $\gamma$-ray spectra of the diffuse emission and HESS J1745-290. The $Y$ axis shows fluxes multiplied by a factor $\mathrm{E}^{2}$, where $\mathrm{E}$ is the energy on the $\mathrm{X}$ axis, in units of $\mathrm{TeVcm}^{-2} \mathrm{~s}^{-1}$. The vertical and horizontal error bars show the $1 \sigma$ statistical error and bin size, respectively. Arrows represent $2 \sigma$ flux upper limits. The $1 \sigma$ confidence bands of the best-fit spectra of the diffuse and HESS J1745-290 are shown in red and blue shaded areas, respectively. Spectral parameters are given in Methods. The red lines show the numerical computations assuming that $\gamma$-rays result from the decay of neutral pions produced by proton-proton interactions. The fluxes of the diffuse emission spectrum and models are multiplied by 10 . 


\section{References}

1. Berezinskii, V. S., Bulanov, S. V., Dogiel, V. A., \& Ptuskin, V. S. Astrophysics of cosmic rays. Amsterdam: North-Holland, edited by Ginzburg, V.L., 1990

2. Malkov, M. A., \& Drury, L. O. Nonlinear theory of diffusive acceleration of particles by shock waves. Reports on Progress in Physics 64, 429-481 (2001)

3. Hillas, A. M. Evolution of ground-based gamma-ray astronomy from the early days to the Cherenkov Telescope Arrays. Astropart. Phys. 43, 19-43 (2013)

4. Aharonian, F. A. Gamma rays from supernova remnants Astropart. Phys. 43, 71-80 (2013)

5. Clavel, M., Terrier, R., Goldwurm, A., Morris, M. R., Ponti, G., Soldi, S., and Trap, G., Echoes of multiple outbursts of Sagittarius A* revealed by Chandra Astron. Astrophys. 558, A32 (2013)

6. Su, M., Slatyer, T. R., \& Finkbeiner, D. P. Giant Gamma-ray Bubbles from Fermi-LAT: Active Galactic Nucleus Activity or Bipolar Galactic Wind? Astrophys. J., 724, 1044-1082 (2010)

7. Aharonian, F., et al. [H.E.S.S. Collaboration], Discovery of very-high-energy gamma-rays from the Galactic centre ridge. Nature 439, 695-698 (2006).

8. Yang, R., Jones, D. I., \& Aharonian, F., Fermi-LAT observations of the Sagittarius B complex. Astron. Astrophys. 580, A90 (2015).

9. Aharonian, F., Very high energy cosmic gamma radiation : a crucial window on the extreme Universe, River Edge, NJ: World Scientific Publishing, 2004

10. Strong, A. W., Moskalenko, I. V. \& Ptuskin, V. S., Cosmic-Ray Propagation and Interactions in the Galaxy. ARNPS, 57, 285-327 (2007).

11. Aharonian, F., et al. [HESS Collaboration], Very high-energy gamma rays from the direction of Sagittarius A*. Astron. Astrophys. 425, L13-L17 (2004).

12. Kosack, K. et al. [VERITAS Collaboration], TeV Gamma-Ray Observations of the Galactic Center. Astrophys. J. Lett. 608, L97-L100 (2004).

13. Tsuchiya, K., et al. [CANGAROO-II Collaboration], Detection of Sub-TeV Gamma Rays from the Galactic Center Direction by CANGAROO-II. Astrophys. J. Lett., 606, L115-L118 (2004).

14. Albert, J., et al. [MAGIC Collaboration], Observation of Gamma Rays from the Galactic Center with the MAGIC Telescope. Astrophys. J. Lett., 638, L101-L104 (2006).

15. Aharonian, F. \& Neronov, A., High-Energy Gamma Rays from the Massive Black Hole in the Galactic Center. Astrophys. J. 619, 306-313 (2005). 
16. Wang, Q. D., Lu, F. J. \& Gotthelf, E. V. G359.95-0.04: pulsar candidate near Sgr A*. Mon. Not. R. Astron. Soc. 367, 937-944 (2006).

17. Hinton, J. A. \& Aharonian, F., Inverse Compton Scenarios for the TeV Gamma-Ray Emission of the Galactic Centre. Astrophys. J. 657, 302-307 (2007).

18. Belikov, A. V., Zaharijas, G. \& Silk, J. Study of the gamma-ray spectrum from the Galactic Center in view of multi-TeV dark matter candidates. Phys. Rev. D. 86, 083516 (2012).

19. Aharonian, F., et al. [H.E.S.S. Collaboration], Spectrum and variability of the Galactic Center VHE gamma-ray source HESS J1745-290. Astron. Astrophys. 503, 817-825 (2009).

20. Archer, A., et al. [VERITAS Collaboration], Very-high Energy Observations of the Galactic Center Region by VERITAS in 2010-2012. Astrophys. J., 790, 149 (2014).

21. Acero, F., et al. [HESS Collaboration], Localising the VHE gamma-ray source at the Galactic Centre. Mon. Not. R. Astron. Soc. 402, 1877-1882 (2010).

22. Crocker, R. M., Jones, D. I., Aharonian, F., Law, C.J., Melia, F. \& Ott, J., $\gamma$-rays and the farinfrared-radio continuum correlation reveal a powerful Galactic Centre wind. MNRAS Lett., 411, L11-L15 (2011).

23. Bykov, A.M., Nonthermal particles and photons in starburst regions and superbubbles. Astron. Astrophys. Rev. 22, 77 (2014).

24. Bell, A., Schure, K., Reville, B. \& Giacinti, G. Cosmic ray acceleration and escape from supernova remnants. Mon. Not. R. Astron. Soc. 431, 415-429 (2013).

25. Istomin, Y. N., On the origin of galactic cosmic rays. New Astronomy, 27, 13-18 (2014).

26. Atoyan, A. \& Dermer, C. D., TeV Emission from the Galactic Center Black Hole Plerion. Astrophys. J. Lett. 617, L123-L126 (2004).

27. Genzel, R., Eisenhauer, F. \& Gillessen, S., The Galactic Center massive black hole and nuclear star cluster. Rev. Mod. Phys. 82, 3121-3195 (2010).

28. Cristofari, P., Gabici, S., Casanova, S., Terrier, R. \& Parizot, E., Acceleration of cosmic rays and gamma-ray emission from supernova remnants in the Galaxy. Mon. Not. Roy. Astron. Soc. 434, 2748-2760 (2013).

29. E. Parizot, Cosmic Ray Origin: Lessons from Ultra-High-Energy Cosmic Rays and the Galactic/Extragalactic Transition. Nucl. Phys. Proc. Suppl. 256, 197-212 (2014).

30. Tsuboi, M., Handa, T. \& Ukita, N., Dense Molecular Clouds in the Galactic Center Region. I. Observations and Data. Astrophys. J. Supp. 120, 1-39 (1999). 
Acknowledgements The support of the Namibian authorities and of the University of Namibia in facilitating the construction and operation of H.E.S.S. is gratefully acknowledged, as is the support by the German Ministry for Education and Research (BMBF), the Max Planck Society, the German Research Foundation (DFG), the French Ministry for Research, the CNRS-IN2P3 and the Astroparticle Interdisciplinary Programme of the CNRS, the U.K. Science and Technology Facilities Council (STFC), the IPNP of the Charles University, the Czech Science Foundation, the Polish Ministry of Science and Higher Education, the South African Department of Science and Technology and National Research Foundation, and by the University of Namibia. We appreciate the excellent work of the technical support staff in Berlin, Durham, Hamburg, Heidelberg, Palaiseau, Paris, Saclay, and in Namibia in the construction and operation of the equipment.

Author Contributions F. Aharonian, S. Gabici, E. Moulin and A.Viana have analysed and interpreted the data, and prepared the manuscript. The whole H.E.S.S. collaboration has contributed to the publication with involvement at various stages ranging from the design, construction and operation of the instrument, to the development and maintenance of all software for data handling, data reduction and data analysis. All authors have reviewed, discussed, and commented on the present results and on the manuscript.

Author Information Reprints and permissions information is available at www.nature.com/reprints. The authors declare no competing financial interests. Correspondence and requests for materials should be addressed HESS Collaboration (contact.hess@ hess-experiment.eu). 


\section{Methods}

\section{Data and spectral analyses}

The phase-I of the H.E.S.S. array consists of four identical imaging atmospheric Cherenkov telescopes. The study presented in the Letter makes use of data collected from 2004 to 2013. Data are taken in wobble mode where the pointing direction is chosen at an alternating offset of $0.7^{\circ}$ to $1.1^{\circ}$ from the target position ${ }^{31}$. Standard quality selection is applied to the data ${ }^{311}$, and after the selection procedure the dataset amounts to 226 hours of live time at the nominal position of Sgr $A^{*}$. The analysis technique used to select the $\gamma$-ray events is based on a semi-analytical model of the air shower development ${ }^{32}$. The background level is calculated in each position using the Ring Background method ${ }^{33}$. The map shown in Fig. 1 is the $\gamma$-ray excess count map per $0.02 \times 0.02 \mathrm{deg}^{2}$ smoothed by the H.E.S.S. point spread function and corrected for the telescope radial acceptance.

The spectral reconstruction is based on a forward-folding method ${ }^{\sqrt{34}}$. This method is based on a maximum-likelihood procedure, comparing the energy distributions of signal and background events to predefined spectral shapes. The energy spectra (Fig. 3) are both fitted by a power-law, $d N / d E=\Phi_{1}(E / \mathrm{TeV})^{-\Gamma_{1}}$, where $\Phi_{1}$ is the flux normalisation and $\Gamma_{1}$ is the spectral index, and by a power-law with an exponential cutoff, $d N / d E=\Phi_{0}(E / \mathrm{TeV})^{-\Gamma_{0}} \times \exp \left(-E / E_{\text {cut }}\right)$, where $\Phi_{0}$ is the flux normalisation, $\Gamma_{0}$ is the spectral index and $E_{\text {cut }}$ is the cut-off energy. A likelihood-ratio test between these two representations is performed to determine whether a significant deviation from a pure power-law is preferred by the data. The best-fit spectra together with their $1 \sigma$ confidencelevel band are shown in Fig. 3 (shaded red and blue regions). The spectral points are uncorrelated flux points, obtained from the reconstructed best-fit spectrum. The error bars are the $1 \sigma$ Poisson deviation of the excess number of events in the energy bin.

The diffuse emission spectrum is extracted from an annulus centered at Sgr A* (right panel of Fig. 1) with inner and outer radii of $0.15^{\circ}$ and $0.45^{\circ}$, respectively, and a solid angle of $1.4 \times$ $10^{-4}$ sr. The best-fit spectrum is given by a power-law with $\Phi_{1}=\left(1.92 \pm 0.08_{\text {stat }} \pm 0.28_{\text {syst }}\right) \times$ $10^{-12} \mathrm{TeV}^{-1} \mathrm{~cm}^{-2} \mathrm{~s}^{-1}$, and a photon index $\Gamma_{1}=2.32 \pm 0.05_{\text {stat }} \pm 0.11_{\text {syst }}$. Its $\gamma$-ray luminosity above $1 \mathrm{TeV}$ is $L_{\gamma}(\geq 1 \mathrm{TeV})=\left(5.69 \pm 0.22_{\text {stat }} \pm 0.85_{\text {syst }}\right) \times 10^{34} \mathrm{erg} \mathrm{s}^{-1}$. The fit of a power-law with an exponential energy cutoff is not preferred by the data. When compared with a pure powerlaw the likelihood-ratio test gives a p-value of $0.8(\sim 0.25$ standard deviations from the power-law fit). In order to investigate the possibility of spatial variations of the spectral indices over the central molecular zone, the spectra within all the regions (left panel of Fig. 1) are reconstructed. All the indices are compatible, within a $1 \sigma$ standard deviation, to an index of $2.32 \pm 0.05_{\text {stat }} \pm 0.10_{\text {syst }}$ (see Extended Data Table 3). The spectrum of the central source is extracted from a circular region of radius $0.1^{\circ}$ centered on Sgr A*. The best-fit spectrum is a power-law with an exponential cutoff with $\Phi_{0}=\left(2.55 \pm 0.04_{\text {stat }} \pm 0.37_{\text {syst }}\right) \times 10^{-12} \mathrm{TeV}^{-1} \mathrm{~cm}^{-2} \mathrm{~s}^{-1}$, a photon index of $\Gamma_{0}=$ $2.14 \pm 0.02_{\text {stat }} \pm 0.10_{\text {syst }}$, and an energy cutoff at $E_{\text {cut }}=\left(10.7 \pm 2.0_{\text {stat }} \pm 2.1_{\text {syst }}\right) \mathrm{TeV}$. When compared with a pure power-law the likelihood-ratio test gives a p-value of $3 \times 10^{-5}$. A power-law with an exponential cutoff is clearly preferred by the data.

The diffuse $\gamma$-ray spectrum from the decay of neutral pions produced by $p p$ interactions re- 
flects the parent proton spectrum. The normalisation of the proton spectrum depends on a combination of the injection power, target mass and propagation effects. Its shape, however, is completely defined by the observed $\gamma$-ray spectrum. In the case of a $\gamma$-ray spectrum following a power-law with index $\Gamma_{1}$, the parent proton spectrum should follow a power-law with an index $\Gamma_{\mathrm{p}} \approx \Gamma_{1}+0.1^{9}$. Using the parametrization of ref. 39, the best-fit proton spectrum to the H.E.S.S. data is obtained for a power-law with $\Gamma_{\mathrm{p}} \approx 2.4$. The corresponding $1 \sigma$ confidence band of this $\gamma$-ray spectrum is described by the red shaded area in Fig. 3. A fit to the data is also done with the $\gamma$-ray spectral shape derived from a proton spectrum following a power-law with an exponential cutoff. When compared with a power-law the likelihood-ratio test gives a p-value of $0.9(\sim 0.12$ standard deviations from the power-law fit), and it is thus not preferred by the data. The lower limits on the proton spectrum energy cut-off can thus be derived from this fit. The $68 \%, 90 \%$ and $95 \%$ confidence level deviation from the H.E.S.S. data is found for proton spectra following a power-law with $\Gamma_{\mathrm{p}} \approx 2.4$ and cutoffs at $2.9 \mathrm{PeV}, 0.6 \mathrm{PeV}$ and $0.4 \mathrm{PeV}$, respectively. Their corresponding $\gamma$-ray spectra are plotted in Fig. 3 (red long-dashed, dashed and dotted lines, respectively). The numerical computation of the energy spectrum of cosmic-ray protons with energy $>10 \mathrm{TeV}$ escaping from the central source at a rate of $\sim 8 \times 10^{37} \mathrm{erg} / \mathrm{s}$ over a time $\sim 6 \times 10^{3} \mathrm{yr}$, is shown in Fig. 3 (red solid line). Their injection spectrum is $Q(E) \propto E^{-2.2}$ extending up to $4 \mathrm{PeV}$, and their transport is described by a diffusion coefficient $D(E)=6 \times 10^{29}(E / 10 \mathrm{TeV})^{\beta} \mathrm{cm}^{2} / \mathrm{s}$, with $\beta=0.3$. The resulting $\gamma$-ray spectrum is well compatible with the best-fit diffuse spectrum.

\section{Mass estimation and cosmic-ray energy density in the Central Molecular Zone}

The derivation of the cosmic-ray density profile in the central molecular zone rests on the distribution of target material (for cosmic-ray interactions). The bulk of the gas in the Galactic Centre region is in the form of the molecular hydrogen $(\mathrm{H} 2)$, which is very difficult to detect directly. Therefore indirect methods of estimating the mass using tracer molecules must be applied. Tracer molecules are typically rare relative to $\mathrm{H} 2$ but much easier to detect and with an approximately known ratio to $\mathrm{H} 2$. The mass estimates used in this paper are based on the line emission of the CS molecule at the $\mathrm{J}=1-0$ transition ${ }^{30}$. In order to evaluate the systematic uncertainties in these estimates, other channels, such as the line emission from transitions of ${ }^{12} \mathrm{C}^{16} \mathrm{O}^{36}$ and $\mathrm{HCN} \mathrm{N}^{\sqrt{37}}$ molecules are also invoked. The total mass in the inner $150 \mathrm{pc}$ of the central molecular zone is estimated $\left(3_{-1}^{+2}\right) \times 10^{7} \mathrm{M}_{\odot}{ }^{30,35}$. The regions showed in Fig. 1 (left) almost completely cover the inner $150 \mathrm{pc}$ of the central molecular zone and are used to extract the radial distribution of cosmic rays. They are symmetrically distributed around the centre of the central molecular zone, which is offset from $\mathrm{Sgr} \mathrm{A}^{*}$ by $\sim 50 \mathrm{pc}$ in positive Galactic longitudes $(1 \sim 0.33 \mathrm{deg})$. These regions are described here below:

Ring 1 (R1) : Semi-annulus with $\left[r_{\text {in }}, r_{\text {out }}\right]=\left[0.1^{\circ}, 0.15^{\circ}\right]$, where $r_{\text {in }}$ and $r_{\text {out }}$ are the inner and outer radii, respectively. A section of $66^{\circ}$ is excluded in order to avoid a newly detected source which will be reported elsewhere. This section is bounded by the opening angles of $+10^{\circ}$ and $-56^{\circ}$ from the positive Galactic longitude axis (see Fig. 1). The average radial distance from Sgr A* is $r_{d}=18.5$ pc. 
Ring 2 (R2) : Semi-annulus $\left[\mathrm{r}_{\text {in }}, \mathrm{r}_{\text {out }}\right]=\left[0.15^{\circ}, 0.2^{\circ}\right], \mathrm{r}_{d}=25.9 \mathrm{pc}$.

Ring $3(\mathbf{R 3})$ : Semi-annulus $\left[\mathrm{r}_{\mathrm{in}}, \mathrm{r}_{\text {out }}\right]=\left[0.2^{\circ}, 0.3^{\circ}\right], \mathrm{r}_{d}=37.1 \mathrm{pc}$.

Circle 1/1b $(\mathbf{C 1} / \mathbf{C 1 b})$ : circular region with $0.1^{\circ}$ of radius centred at $1=0.344^{\circ} / 359.544^{\circ}, \mathrm{b}=$ $-0.04588^{\circ}$, in galactic coordinates, and $\mathrm{r}_{d}=59.3 \mathrm{pc}$

Circle 2/2b (C2/C2b): circular region with $0.1^{\circ}$ of radius centred at $1=0.544^{\circ} / 359.344^{\circ}, \mathrm{b}=$ $-0.04588^{\circ}, \mathrm{r}_{d}=89.0 \mathrm{pc}$

Circle $3(\mathbf{C 3})$ : circular region with $0.1^{\circ}$ of radius centred at $\mathrm{l}=0.744^{\circ}, \mathrm{b}=-0.04588^{\circ}, \mathrm{r}_{d}=118.6$ $\mathrm{pc}$

Circle 4 (C4): circular region with $0.1^{\circ}$ of radius centred at $\mathrm{l}=0.944^{\circ}, \mathrm{b}=-0.04588^{\circ}, \mathrm{r}_{d}=148.3$ $\mathrm{pc}$

Circle 5 (C5): circular region with $0.1^{\circ}$ of radius centred at $\mathrm{l}=1.144^{\circ}, \mathrm{b}=-0.04588^{\circ}, \mathrm{r}_{d}=178.0$ $\mathrm{pc}$

If the $\gamma$-ray emission is completely due to the decay of neutral pions produced in protonproton interactions, then the $\gamma$-ray luminosity $L_{\gamma}$ above the energy $E_{\gamma}$ is related to the total energy of cosmic-ray protons $W_{p}$ as

$$
L_{\gamma}\left(\geq E_{\gamma}\right) \sim \eta_{N} \frac{W_{p}\left(\geq 10 E_{\gamma}\right)}{t_{p p \rightarrow \pi^{0}}}
$$

where $t_{p p \rightarrow \pi^{0}}=1.6 \times 10^{8} \mathrm{yrs}\left(1 \mathrm{~cm}^{-3} / n_{\mathrm{H}}\right)$ is the proton energy loss timescale due to neutral pion production in an environment of hydrogen gas of density $n_{\mathrm{H}}$, and $\eta_{N} \approx 1.5$ accounts for the presence of nuclei heavier than hydrogen in both cosmic rays and interstellar matter. The energy density of cosmic rays $w_{\mathrm{CR}}$, averaged along the line of sight is then:

$$
w_{\mathrm{CR}}\left(\geq 10 E_{\gamma}\right)=\frac{W_{p}\left(\geq 10 E_{\gamma}\right)}{V} \sim 1.8 \times 10^{-2}\left(\frac{\eta_{N}}{1.5}\right)^{-1}\left(\frac{L_{\gamma}\left(\geq E_{\gamma}\right)}{10^{34} \mathrm{erg} / \mathrm{s}}\right)\left(\frac{M}{10^{6} M_{\odot}}\right)^{-1} \mathrm{eV} / \mathrm{cm}^{3},
$$

where $M$ is the mass of the relevant region. The $\gamma$-ray luminosity above $1 \mathrm{TeV}$ and the mass estimates (based on three tracers) for all regions are presented in Extended Data Table 1. The cosmic-ray energy densities in different regions, given in units of $10^{-3} \mathrm{eV} / \mathrm{cm}^{3}$, which is the value of the local cosmic-ray energy density $w_{0}(\geq 10 \mathrm{TeV})$ (as measured in the Solar neighbourhood), are presented in Extended Data Table 2.

The uncertainty in the cosmic-ray energy density comes basically from the uncertainty in the mass estimates. The independent estimates from different tracers result in the cosmic-ray enhancement factors in the inner regions of the central molecular zone ( $r \leq 25 \mathrm{pc}$ from the Galactic Centre) $16_{-5}^{+10}(\mathrm{CS}), 22_{-7}^{+14}(\mathrm{CO})$ and $24_{-8}^{+16}(\mathrm{HCN})$. The cosmic-ray radial distribution is also computed for all the different channels. The results (shown in Fig.2 for CS line tracer) are in good 
agreement with the $1 / r$ profile with $\chi^{2} /$ d.o.f. $=11.8 / 9, \chi^{2} /$ d.o.f. $=9.4 / 9$ and $\chi^{2} /$ d.o.f. $=11.0 / 8$ for mass estimates based on the CS, $\mathrm{CO}$ and $\mathrm{HCN}$ tracers, respectively. At the same time, the data are in obvious conflict with the $w(r) \propto 1 / r^{2}, \chi^{2} /$ d.o.f. $=73.2 / 9(\mathrm{CS}), \chi^{2} /$ d.o.f. $=78.0 / 9(\mathrm{CO})$ and $\chi^{2} /$ d.o.f. $=57.9 / 8(\mathrm{HCN})$, and $w(r) \propto$ constant profiles, $\chi^{2} /$ d.o.f. $=61.2 / 9(\mathrm{CS}), \chi^{2} /$ d.o.f. $=$ 45.6/9 (CO) and $\chi^{2} /$ d.o.f. $=77.1 / 8(\mathrm{HCN})$. Finally, when fitting a $1 / r^{\alpha}$ profile to the data, the best fit is found for $\alpha$ equal to $1.10 \pm 0.12(\mathrm{CS}), 0.97 \pm 0.13(\mathrm{CO})$ and $1.24 \pm 0.12(\mathrm{HCN})$, with $\chi^{2} /$ d.o.f. $=11.1 / 8, \chi^{2} /$ d.o.f. $=9.34 / 8$ and $\chi^{2} /$ d.o.f. $=6.5 / 7$, respectively, which confirms the preference for an $1 / r$ density profile to describe the data.

\section{Spectral analysis within the central molecular zone}

The findings of a PeVatron accelerating protons in a quasi-continuous regime, over a sufficiently long period of time to fill the whole central molecular zone, implies that the gamma-ray energy spectral shape should be spatially independent over the central molecular zone. The available statistics in each of these regions prevent us to test the existence of a cutoff beyond $10 \mathrm{TeV}$. The indices of all the regions are presented in Extended Data Table 3. All the indices are compatible within a $1 \sigma$ standard deviation to an index of $2.32 \pm 0.05_{\text {stat }} \pm 0.10_{\text {syst }}$, as measured in the annulus in the right panel of Fig. 1, and used to derive the properties of the Galactic Centre PeVatron. The compatibility of the measured spectral indices over the $200 \mathrm{pc}$ of the central molecular zone provides an additional piece of evidence for the scenario proposed in this Letter.

\section{Multi-TeV $\gamma$-rays of leptonic origin?}

For the diffuse $\gamma$-ray emission of Galactic Centre we deal with quite specific conditions which strongly constrain the possible scenarios of $\gamma$-ray production. Two major radiation mechanisms are related to interactions of ultrarelativistic protons and electrons, with the dense gas in the central molecular zone and with the ambient infrared radiation fields, respectively. For explanation of multi-TeV $\gamma$-rays, the maximum energy of protons and electrons should be as large as $\sim 1 \mathrm{PeV}$ and $\sim 100 \mathrm{TeV}$, respectively. Additionally, these particles should effectively propagate and fill the entire central molecular zone. While in the case of the hadronic scenario one needs to postulate an existence of a PeVatron in the Galactic Centre, any 'leptonic' model of $\gamma$-ray production should address the following questions: (i) whether the accelerator could be sufficiently effective to boost the energy of electrons up to $\geq 100 \mathrm{TeV}$ under the severe radiative losses in the Galactic Centre; (2) whether these electrons can escape the sites of their production and propagate over distances of tens of parsecs; (3) whether they can explain the observed hard spectrum of multi-TeV $\gamma$-rays.

Acceleration of electrons to multi-100 $\mathrm{TeV}$ energies is more difficult than acceleration of protons because of severe synchrotron and inverse Compton (IC) losses. Formally, acceleration of electrons to energies beyond $100 \mathrm{TeV}$ is possible in the so-called extreme accelerators, where the acceleration proceeds at the maximum possible rate allowed by classical electrodynamics, $t_{\text {acc }} \sim$ $R_{\mathrm{L}} / c \approx 0.4(E / 100 \mathrm{TeV})(\mathrm{B} / 1 \mu \mathrm{G})^{-1} \mathrm{yr}$, where $R_{\mathrm{L}}$ is the Larmor radius. Even so, the escape of such energetic electrons from the accelerator and their propagation far enough (tens of parsecs) 
to fill the central molecular zone, can be realised only for rather unrealistically weak magnetic fields and fast diffusion. Indeed, the propagation time over a distance $R$ and for a particle diffusion coefficient $D$ is equal to $t_{\text {diff }}=R^{2} / 6 D \sim 2 \times 10^{3}(R / 200 \mathrm{pc})^{2}\left(D / 10^{30} \mathrm{~cm}^{2} / \mathrm{s}\right) \mathrm{yr}$ and, for typical interstellar conditions, is much longer than the synchrotron loss time of electrons with energy $E_{e}$, $t_{\text {synch }} \approx 10(B / 100 \mu \mathrm{G})^{-2}\left(\mathrm{E}_{\mathrm{e}} / 100\right)^{-1} \mathrm{yr}$.

The efficiency of a given $\gamma$-ray emitting process is determined by the cooling time of particles through that specific channel compared to the characteristic times of other (radiative and nonradiative) processes. The cooling times of relativistic electrons in the central molecular zone are shown in Extended Data Fig. 1. While bremsstrahlung and IC scattering result in $\gamma$-ray emission, the ionisation and synchrotron losses reduce the efficiency of $\gamma$-ray production. Bremsstrahlung is an effective mechanism of $\gamma$-radiation at $\mathrm{GeV}$ energies. Above $100 \mathrm{GeV}$ the IC cooling becomes more effective ( $t_{\mathrm{IC}}<t_{\mathrm{br}}$; where $t_{\mathrm{IC}}$ and $t_{\mathrm{br}}$ are the electrons' cooling times through IC and bremsstrahlung, respectively), and strongly dominates over bremsstrahlung at energies above 10 TeV. This can be seen in Extended Data Fig. 2 where the results of calculations of the spectral energy distribution of broad-band emission of electrons are shown. The calculations are performed for an acceleration spectrum following a power-law with an exponential cutoff at $100 \mathrm{TeV}$. Assuming that electrons are injected in a continuous regime, the steady-state spectrum of electrons is obtained by solving the kinetic equation which takes into account the energy losses of electrons due to ionisation, bremsstrahlung, synchrotron radiation and IC scattering. At low energies more important are the losses due to the diffusive escape of electrons from the central molecular zone. Although it has been shown that the magnetic field in the Galactic Centre should have a lower-limit of $B=50 \mu \mathrm{G}$ on $400 \mathrm{pc}$ scales $^{44}$, here we assume a very low $B=15 \mu \mathrm{G}$. Even with such low magnetic field, it is seen that above $10 \mathrm{TeV}$ the calculations do not match the observed fluxes. Formally, one can assume, higher, e.g. by an order of magnitude, gas density (e.g. if $\gamma$-rays are produced mainly in dense cores of molecular clouds), thus the bremsstrahlung would dominate over the IC contribution, and the flux of $\gamma$-rays could be increased. However, for any reasonable magnetic field, the synchrotron losses above $10 \mathrm{TeV}$ will dominate over bremsstrahlung. This will make the steady-state electron spectrum steeper with power-law index $\alpha=\alpha_{0}+1$ ( $\alpha_{0}$ is the power-law index of the electron injection spectrum). Since the $\gamma$-ray spectrum produced due to bremsstrahlung mimics the energy spectrum of parent electrons $(\Gamma=\alpha)$, at energies of $\gamma$-rays above a few $\mathrm{TeV}$ we should expect quite steep spectrum of $\gamma$-rays with a power-law index $\Gamma>3.4$. This is in apparent conflict with observations.

\section{Multiwavelength and multi-messenger signatures of PeVatrons}

Galactic PeVatrons have unique signatures which allow their unambiguous identification among other particle accelerators. Such signatures are related to the neutral secondary products resulting from hadronic interactions of accelerated $\mathrm{PeV}$ protons and nuclei $(E \geq 0.1 \mathrm{PeV} /$ nucleon$)$ with the ambient gas. The secondaries produced at low energies, in particular $\mathrm{MeV} / \mathrm{GeV} \gamma$-rays and the radio synchrotron emission of primary and secondary electrons and positrons, do carry information about the accelerator, however, strictly speaking, they are not directly linked to the 
$\mathrm{PeV}$ particles. The extrapolations from low to high energies based on theoretical assumptions are model-dependent, and therefore biased. Obviously, they cannot substitute the direct measurements at highest energies.

All three products of interactions of ultrarelativistic protons - $\gamma$-rays, neutrinos and electrons - generated through the production and decay of $\pi^{0}, \pi^{+}$and $\pi^{-}$mesons, receive approximately $10 \%$ of the energy of primary protons, thus multi-TeV secondary neutrals carry univocal information about the primary PeV protons. This concerns, first of all, $\geq 10 \mathrm{TeV} \gamma$-rays, because at such high energies the efficiency of leptonic channels of production of high energy $\gamma$-rays in general, and in the central molecular zone, in particular, is dramatically reduced (see above). The flux sensitivity as well as the angular and energy resolutions achieved by the H.E.S.S. array allow adequate studies of the acceleration sites and the propagation of accelerated protons up to $1 \mathrm{PeV}$ based on the morphological and spectral properties of multi-TeV $\gamma$-rays from the Galactic Centre.

An independent and straightforward proof of the hadronic origin of diffuse $\gamma$-rays from the central molecular zone would be the detection of multi-TeV neutrinos spatially correlated with $\gamma$-rays. In Extended Data Fig. 3 we show the fluxes of high energy neutrinos which should accompany the $\gamma$-ray flux presented in Fig. 3. The calculations are based on the parent proton spectrum derived from $\gamma$-ray data, therefore the only free parameter in these calculations is the high energy cutoff $E_{0}$ in the spectrum of parent protons. The condition for the detection of high energy neutrinos by $\mathrm{km}^{3}$-scale detectors (such as IceCube or KM3Net) can be expressed by a minimum flux of $\gamma$-rays, assuming that both neutrinos and $\gamma$-rays are products of $p p$ interactions. The estimate of detectability of neutrinos is most robust (i.e. less sensitive to the spectral shape) when normalised at a particle energy of $\approx 20 \mathrm{TeV}$. Namely, neutrinos can be detected by a $\mathrm{km}^{3}$ volume detector if the differential flux of accompanying $\gamma$-rays at $20 \mathrm{TeV}$ exceeds $10^{-12} \mathrm{TeV} \mathrm{cm}^{-2} \mathrm{~s}^{-1}$ [38. Since the $\gamma$-ray fluxes (Fig. 3) are quite close to this value, we may conclude that multi-TeV neutrinos from the Galactic Centre can be marginally detected by a $\mathrm{km}^{3}$-scale detector after several years of exposure.

The third complementary channel of information about the $\mathrm{PeV}$ protons is carried by the secondary electrons, through their synchrotron radiation. In $p p$ interactions, electrons are produced in a fair balance with neutrinos and photons (their distribution almost coincides with the spectrum of the electronic neutrinos ${ }^{\sqrt{39}}$, i.e. they carry a significant fraction of the energy of the incident proton. In environments with magnetic field $B \geq 100 \mu \mathrm{G}$, the lifetime of secondary electrons producing $\mathrm{X}$-rays of energy $\epsilon_{x}$ is quite short, $\sim 15(B / 100 \mu \mathrm{G})^{-3 / 2}\left(\epsilon_{\mathrm{x}} / 10 \mathrm{keV}\right)^{-1 / 2} \mathrm{yr}$, compared to other characteristic times, e.g. to the propagation time of protons over the central molecular zone zone. Therefore, hard X-rays can be considered as a "prompt" radiation component emitted in hadronic interactions simultaneously with $\gamma$-rays and neutrinos. The energy release in X-rays calculated self-consistently for the proton spectrum derived from the $\gamma$-ray data, exceeds $10 \%$ of the energy released in multi-TeV neutrinos and $\gamma$-rays (see in Extended Data Fig. 4). In general, this is quite substantial, given the superior flux sensitivity of X-ray instruments, especially for point like sources. However, since the radiation component shown in Extended Data Fig. 4 is integrated over a very large (for $\mathrm{X}$-ray instruments) region with an angular size of $\sim 1^{\circ}$, it is overshadowed by the 
diffuse X-ray emission detected by XMM-Newton ${ }^{40}$. This makes the detection of this component practically impossible.

Finally, one should mention relativistic neutrons as another potential messengers of hadronic processes produced in the Galactic Centre predominantly in the reaction $p p \rightarrow p n \pi^{+}$. However, because of the short lifetime of about $\tau \simeq 10^{3} \mathrm{sec}$, only neutrons of energy $E_{\mathrm{n}} \geq m_{\mathrm{n}} d /(c \tau) \sim$ $10^{18} \mathrm{eV}$ (where $m_{\mathrm{n}}$ is the neutron mass) could reach us before they decay during the "free flight" from the Galactic Centre ( $d=8.5 \mathrm{kpc}$ is the distance to the Galactic Centre). Thus if the proton spectrum in the Galactic Centre extends to extremely high energies, one can probe, in principle, this additional channel of information by detectors of cosmic rays, in particular by the AUGER observatory.

\section{Alternative explanations to the supermassive black hole as the source of cosmic rays responsible for the diffuse $\gamma$-ray emission in the central molecular zone}

Several cosmic-ray sources are present in the Galactic centre region. Besides the Galactic Centre supermassive black hole, discussed in the Letter, alternative sources of the cosmic rays responsible for the $\gamma$-ray emission from the central molecular zone include supernova remnants $(\mathrm{SNRs})^{46}$, stellar clusters ${ }^{22}$, and radio filaments ${ }^{\sqrt{45}}$. Any of these scenarios should satisfy the following conditions, derived from the H.E.S.S. observations of the central molecular zone: $i$ ) the accelerator has to be located in the inner $\sim 10 \mathrm{pc}$ of the Galaxy, ii) the accelerator(s) has(have) to be continuous over a timescale of at least thousands of years, and iii) the acceleration has to proceed up to $\mathrm{PeV}$ energies.

Supernova remnants - The acceleration mechanism operating at SNRs is widely believed to be diffusive shock acceleration, characterized by an acceleration timescale $t_{a c c} \sim D(E) / u_{s}^{2}$, where $D(E) \propto E / B$ is the Bohm diffusion coefficient of a cosmic ray of energy $E$ in a magnetic field $B$ and $u_{s}$ is the shock speed. It is clear, then, that the fastest acceleration rate is obtained for the largest possible values of the shock speed and of the magnetic field strength.

In the early free expansion phase of the SNR evolution, shock speeds as high as $\approx 10^{4} \mathrm{~km} / \mathrm{s}$ can be achieved. The magnetic field strength is also expected to be very large during this early phase (up to $\sim 0.1-1 \mathrm{mG}^{41}$ ) due to efficient field amplification connected to the acceleration of cosmic rays at the shock ${ }^{42}$. Under these circumstances, SNRs can accelerate protons up to an energy of $E_{\text {max }} \approx 10^{14}(B / 100 \mu \mathrm{G})\left(u_{s} / 10000 \mathrm{~km} \mathrm{~s}^{-1}\right)^{2}\left(\Delta t_{\mathrm{PeV}} / \mathrm{yr}\right) \mathrm{eV}$, where $\Delta t_{\mathrm{PeV}}$ is the duration of the phase where shock speed and magnetic field are high enough to allow the acceleration of particles up to $\mathrm{PeV}$ energies. It has been shown in ref. 24 that the duration of this phase is of the order of tens of years (definitely less than a century). Thus, even though SNRs can potentially provide PeV particles, they cannot act as (quasi) continuous injectors of such energetic particles for a time of the order of thousands of years.

Stellar clusters - Other possible cosmic-ray sources in the Galactic Centre region are stellar clusters. Three of them are known in the inner $\sim 0.1^{\circ}-0.2^{\circ}$ region: the central, the Arches, and 
the Quintuplet cluster. The most likely sites for acceleration of particles in stellar clusters are the stellar winds of the massive OB stars that form the cluster, and the shocks of the supernovae which mark the end of the life of these stars. However, in order to accelerate particles up to PeV energies, very large shock velocities, of the order of at least $10000 \mathrm{~km} / \mathrm{s}$ are needed ${ }^{24}$. Velocities of this order can hardly be found in stellar wind termination shocks, and thus SNR shocks following the explosion of cluster member stars remain the best candidates as particle accelerators.

Both the Arches and the Quintuplet clusters are located outside of the inner $\sim 10 \mathrm{pc}$ region ${ }^{23}$, and this disfavours their role as accelerators of the cosmic rays responsible for the diffuse $\gamma$-ray emission. On the contrary, the central cluster is located well within the central $10 \mathrm{pc}$ region, and thus should be considered as a potential candidate for the acceleration of cosmic rays in the central molecular zone. The $\gamma$-ray observations suggest that the cosmic-ray source in the Galactic centre should act (quasi) continuously over time $t_{\text {inj }}$ of a few thousands of years. Given that a SNR can accelerate $\mathrm{PeV}$ particles over a time interval $t_{\mathrm{PeV}}$ of less than a century, we would need at least $\sim 10\left(t_{\mathrm{PeV}} / 100 \mathrm{yr}\right)\left(t_{\mathrm{inj}} / 1000 \mathrm{yr}\right)^{-1}$ supernova explosions happening over the last $t_{\text {inj }}$ within the central cluster. Given the very small size of the region $(\sim 0.4 \mathrm{pc})$, such a large SN explosion rate is unrealistic.

Radio filaments - It has been proposed in ref. 45 that the diffuse $\gamma$-ray emission from the central molecular zone was the result of non-thermal Bremsstrahlung from relativistic electrons s5/47]. $^{45}$. In this scenario, the putative sources of $\gamma$-ray emitting electrons are the elongated radio filaments detected throughout the central molecular zone region ${ }^{45}$. This is in tension with one of the main finding of this paper, i.e. the location of the source of cosmic rays in the inner $\sim 10 \mathrm{pc}$ of the Galaxy. Though the acceleration mechanism is not discussed, filaments are assumed to somehow accelerate electrons and then release them in the interstellar medium. In order to fill the whole central molecular zone region before being cooled by synchrotron and inverse Compton losses, electrons are assumed to propagate ballistically (i.e. at the speed of light without a significant deflection in the magnetic field). This unconventional assumption is made at the expenses of a very large energy requirement: observations can be explained if the energy injection rate of cosmic-ray electrons in the central molecular zone is of the order of $\sim 10^{41} \mathrm{erg} / \mathrm{s}^{45}$. This is a very large injection rate, being comparable to the total luminosity of cosmic-ray protons in the whole Galaxy, and makes this scenario problematic. 


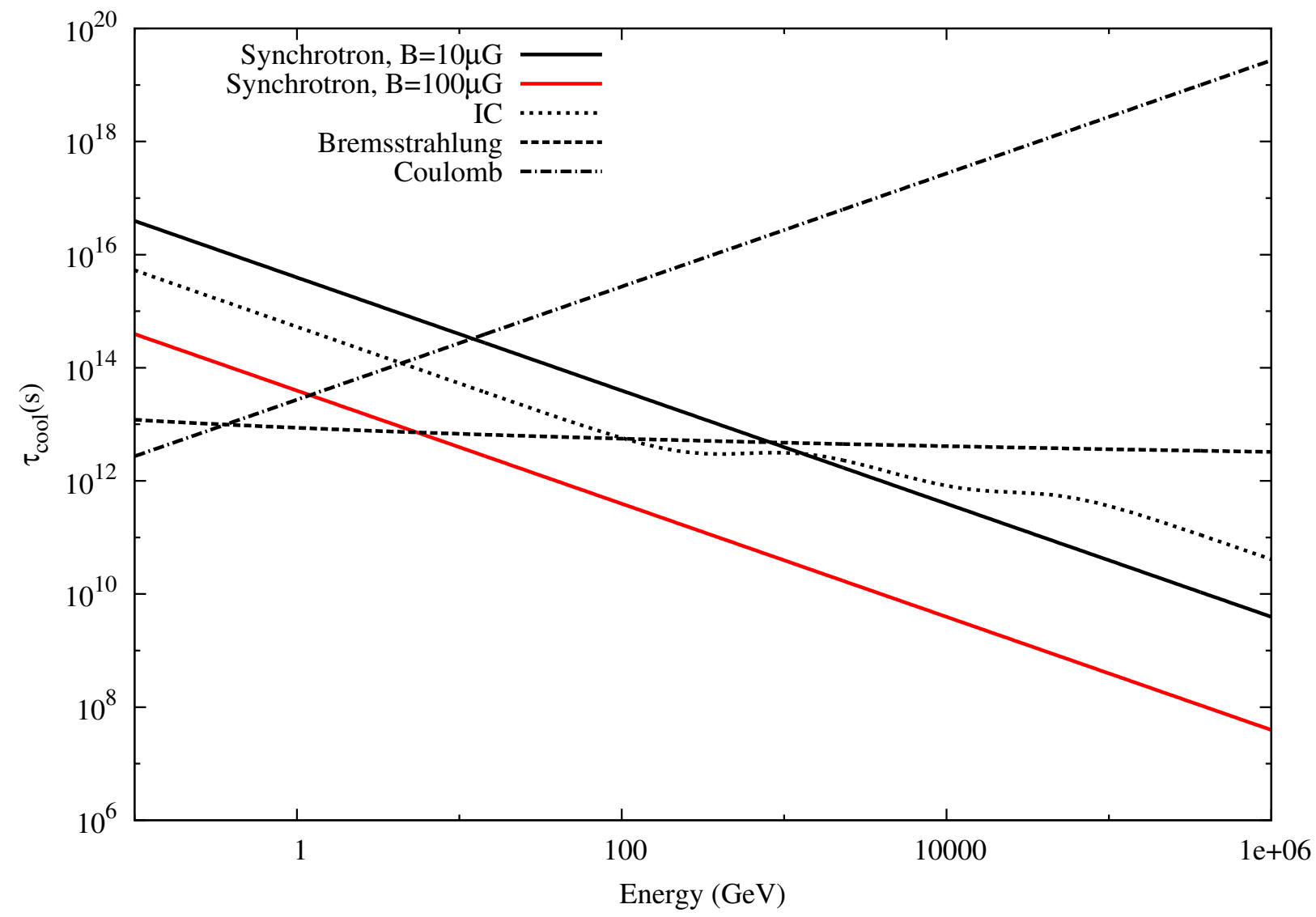

Extended Data Figure 1: Cooling times of electrons in the Galactic Centre as a function of energy. The cooling times due to ionisation (or Coulomb) losses and bremsstrahlung are inversely proportional to the gas density $n$; here $n=100 \mathrm{~cm}^{-3}$ is assumed. The cooling time of the synchrotron radiation is proportional to $1 / B^{2}$, where $\mathrm{B}$ is the magnetic field. The total energy densities of the Cosmic Microwave Background, local near (NIR) and far (FIR) infrared radiation fields used to calculated the cooling time due to the IC scattering are extracted from the GALPROP code ${ }^{43}$. The integrated densities are 17.0 and $1.3 \mathrm{eV} / \mathrm{cm}^{-3}$ for NIR and FIR, respectively. 


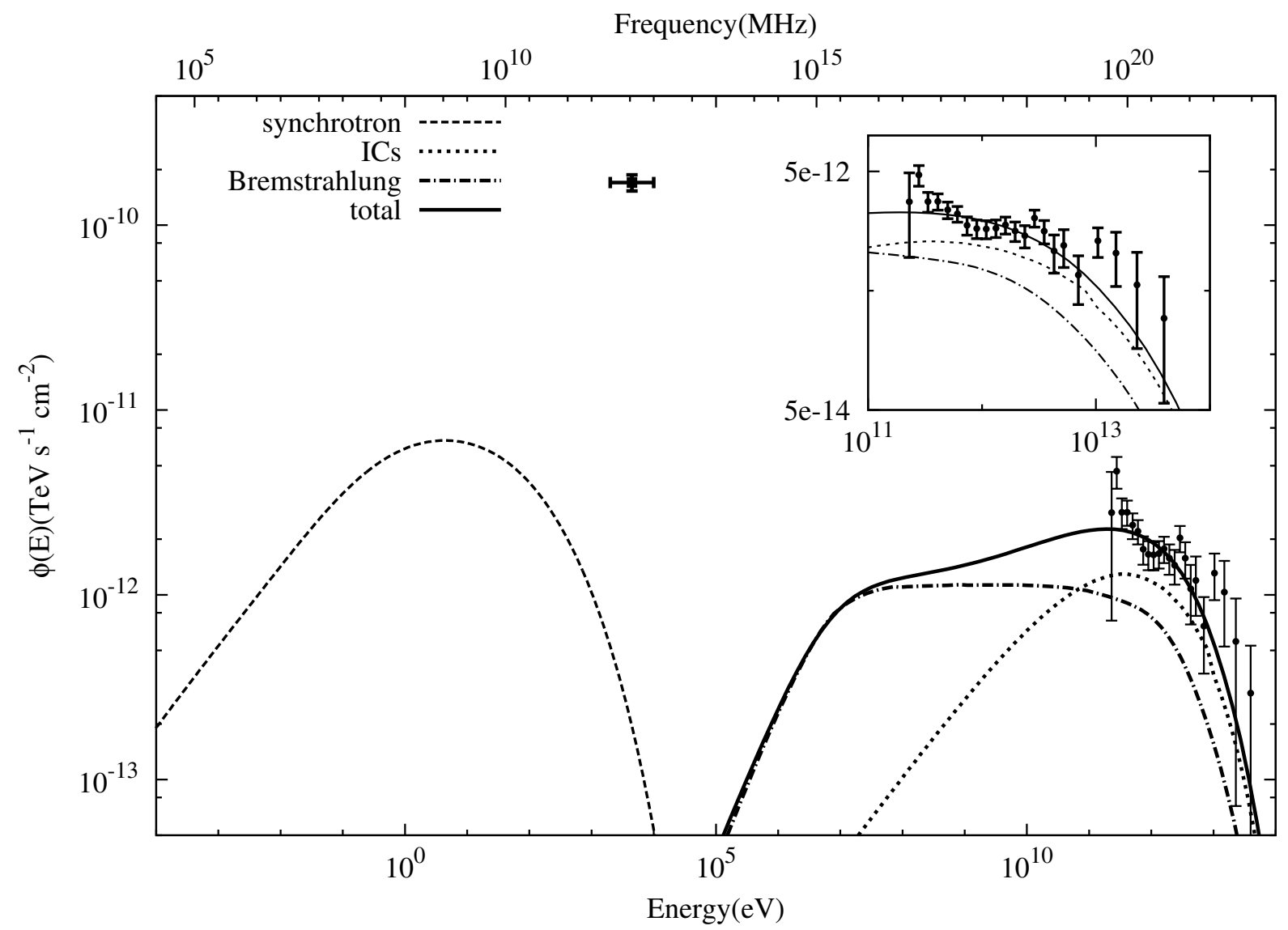

Extended Data Figure 2: Broad-band spectral energy distribution of radiation by relativistic electrons. The flux from synchrotron radiation, bremsstrahlung and the inverse Compton scattering are compared to the fluxes of diffuse $\gamma$-ray emission measured by H.E.S.S. (black points with vertical error bars). The flux of diffuse X-ray emission measured by XMM-Newton ${ }^{40}$ (black point with horizontal error bar) and integrated over the central molecular zone region is also shown. The inset (top right) shows a zoomed view of the SED at the VHE range (100 GeV - 100 $\mathrm{TeV})$. The vertical and horizontal error bars show the $1 \sigma$ statistical errors and the bin size, respectively. $\mu \mathrm{G}$. The flux of diffuse X-ray emission measured by XMM-Newton and integrated over the central molecular zone region is also shown. 


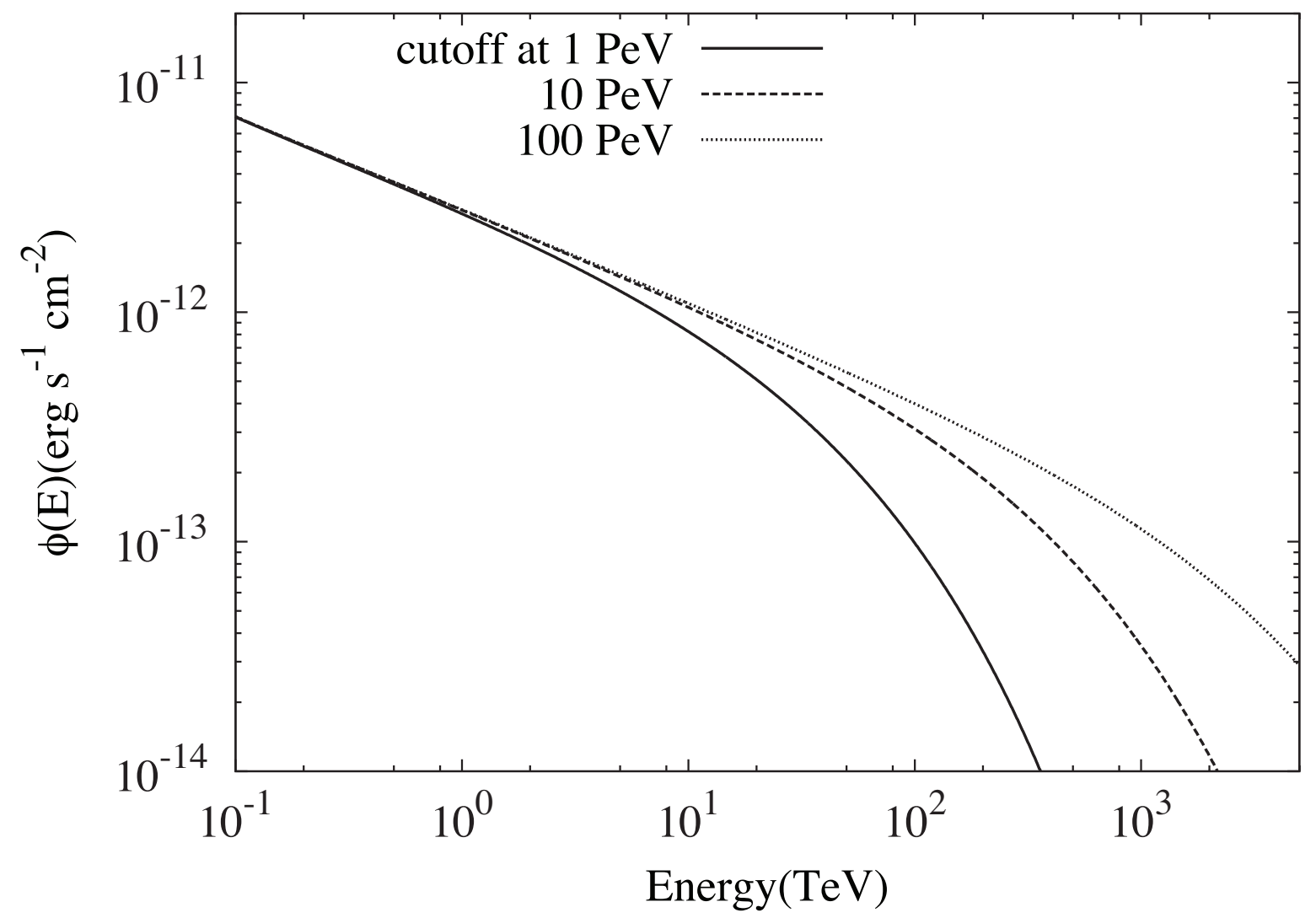

Extended Data Figure 3: The spectral energy distribution of high energy neutrinos - the counterparts of diffuse $\gamma$-rays from the Galactic Centre. The energy spectrum of parent protons is derived from the $\gamma$-ray data. The three curves correspond to different values of the exponential cutoff in the proton spectrum: $1 \mathrm{PeV}, 10 \mathrm{PeV}, 100 \mathrm{PeV}$. 


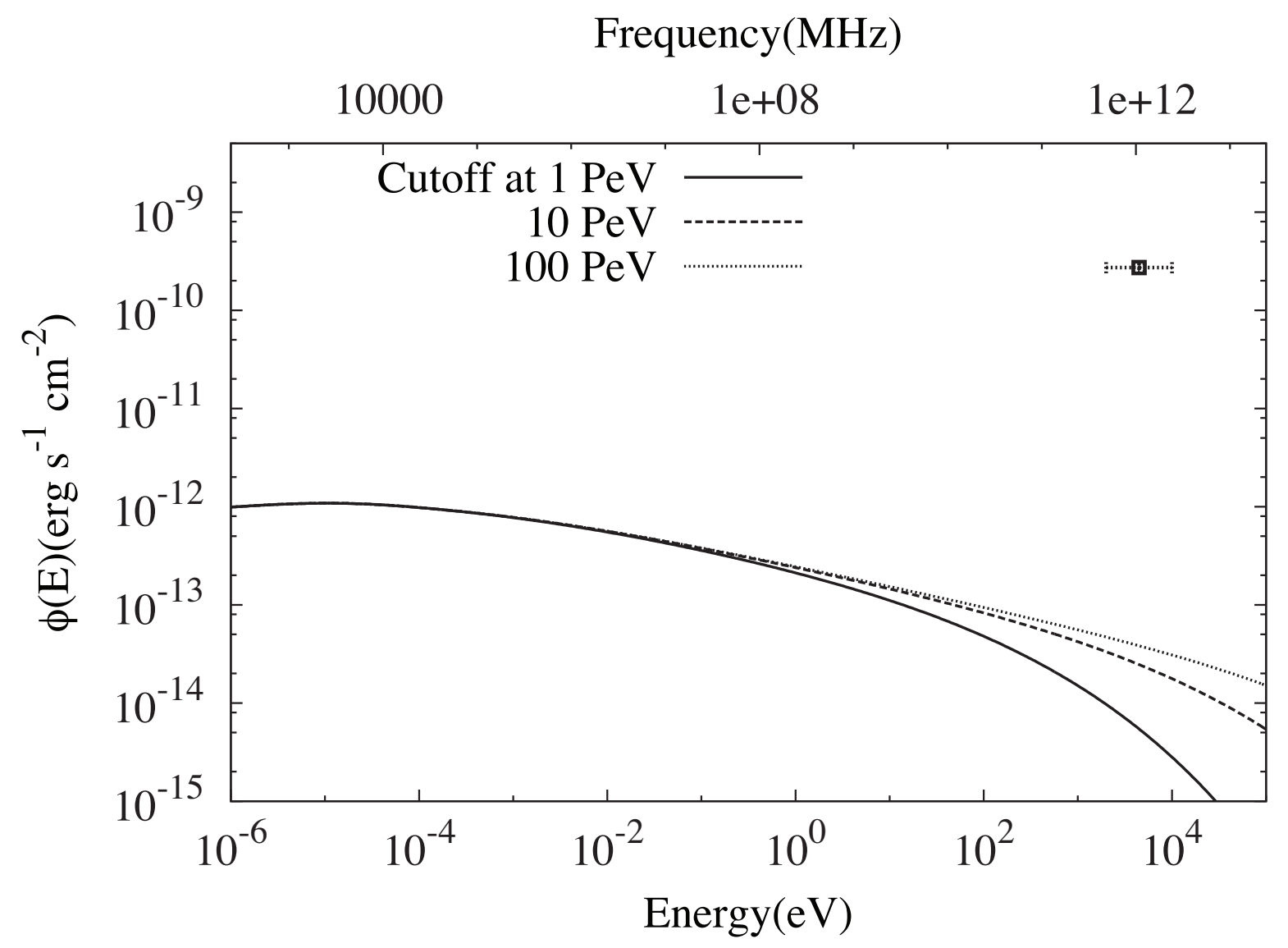

Extended Data Figure 4: The spectral energy distribution of synchrotron radiation of secondary electrons produced in $p p$ interactions. The spectra of protons are the same as in Extended Data Fig. 3. The magnetic field is assumed $100 \mu \mathrm{G}$. The flux of diffuse X-ray emission measured by XMM-Newton and integrated over the central molecular zone region is also shown. The horizontal error bar correspond to the bin size. 


\begin{tabular}{lcccc}
\hline Region & $L_{\gamma}\left(1 \mathrm{TeV} \leq \mathrm{E}_{\gamma}\right)\left[\times 10^{34} \mathrm{erg} \mathrm{s}^{-1}\right]$ & \multicolumn{3}{c}{ Mass $\left[\times 10^{6} \mathrm{M}_{\odot}\right]$} \\
\cline { 3 - 5 } & & CS & CO & HCN \\
\hline R1 & $1.54 \pm 0.06_{\text {stat }} \pm 0.23_{\text {sys }}$ & 1.35 & 0.88 & 0.88 \\
R2 & $1.21 \pm 0.05_{\text {stat }} \pm 0.18_{\text {sys }}$ & 1.11 & 0.98 & 0.86 \\
R3 & $2.05 \pm 0.08_{\text {stat }} \pm 0.30_{\text {sys }}$ & 2.65 & 2.01 & 1.65 \\
C1 & $0.73 \pm 0.03_{\text {stat }} \pm 0.11_{\text {sys }}$ & 1.51 & 1.00 & 1.02 \\
C1b & $0.63 \pm 0.03_{\text {stat }} \pm 0.09_{\text {sys }}$ & 1.49 & 1.03 & 0.82 \\
C2 & $0.84 \pm 0.03_{\text {stat }} \pm 0.12_{\text {sys }}$ & 1.84 & 1.40 & 1.34 \\
C2b & $0.51 \pm 0.02_{\text {stat }} \pm 0.07_{\text {sys }}$ & 1.01 & 0.86 & nc \\
C3 & $0.87 \pm 0.03_{\text {stat }} \pm 0.13_{\text {sys }}$ & 2.69 & 1.55 & 1.73 \\
C4 & $0.42 \pm 0.02_{\text {stat }} \pm 0.06_{\text {sys }}$ & 1.54 & 0.98 & 1.33 \\
C5 & $0.49 \pm 0.02_{\text {stat }} \pm 0.07_{\text {sys }}$ & 1.60 & 1.02 & 1.15 \\
\hline
\end{tabular}

Extended Data Table 1: $\gamma$-ray luminosities and masses in different regions of the central molecular zone. The errors quoted are $1 \sigma$ statistical (stat) and systematical (sys) errors. The region $\mathrm{C} 2 \mathrm{~b}$ does is not covered (nc) in the $\mathrm{HCN}$ line observations $\mathbf{s}^{37}$. 


\begin{tabular}{lccc}
\hline Region & \multicolumn{3}{c}{$w_{\mathrm{CR}}(\geq 10 \mathrm{TeV})\left[10^{-3} \mathrm{eV} / \mathrm{cm}^{3}\right]$} \\
\cline { 2 - 4 } & $\mathrm{CS}$ & $\mathrm{CO}$ & $\mathrm{HCN}$ \\
\hline R1 & $17.3 \pm 2.6$ & $26.7 \pm 4.1$ & $26.6 \pm 4.1$ \\
$\mathrm{R} 2$ & $16.6 \pm 2.5$ & $18.6 \pm 2.8$ & $21.5 \pm 3.3$ \\
$\mathrm{R} 3$ & $11.8 \pm 1.8$ & $15.5 \pm 2.4$ & $18.8 \pm 2.9$ \\
$\mathrm{C} 1$ & $7.3 \pm 1.1$ & $11.0 \pm 1.7$ & $10.9 \pm 1.6$ \\
C1b & $6.5 \pm 1.0$ & $9.3 \pm 1.4$ & $11.6 \pm 1.8$ \\
C2 & $7.0 \pm 1.0$ & $9.1 \pm 1.4$ & $9.4 \pm 1.4$ \\
C2b & $7.7 \pm 1.2$ & $9.0 \pm 1.4$ & $\mathrm{nc}$ \\
C3 & $4.9 \pm 0.7$ & $8.5 \pm 1.3$ & $7.7 \pm 1.1$ \\
C4 & $4.1 \pm 0.6$ & $6.6 \pm 0.9$ & $4.8 \pm 0.7$ \\
C5 & $4.7 \pm 0.7$ & $7.4 \pm 1.1$ & $6.5 \pm 1.0$ \\
\hline
\end{tabular}

Extended Data Table 2: Cosmic-ray energy densities in different regions of the central molecular zone. The densities are given in units of $10^{-3} \mathrm{eV} / \mathrm{cm}^{3}$, which is the value of the local cosmic-ray energy density measured in the Solar neighbourhood. The errors quoted are $1 \sigma$ statistical plus systematical errors. The region $\mathrm{C} 2 \mathrm{~b}$ does is not covered (nc) in the $\mathrm{HCN}$ line observations $\mathrm{s}^{37}$. 


\begin{tabular}{ll}
\hline Region & Power-law spectral index \\
\hline R1 & $2.27 \pm 0.05_{\text {stat }} \pm 0.11_{\text {sys }}$ \\
R2 & $2.33 \pm 0.07_{\text {stat }} \pm 0.11_{\text {sys }}$ \\
R3 & $2.18 \pm 0.07_{\text {stat }} \pm 0.10_{\text {sys }}$ \\
C1 & $2.38 \pm 0.10_{\text {stat }} \pm 0.11_{\text {sys }}$ \\
C1b & $2.26 \pm 0.10_{\text {stat }} \pm 0.10_{\text {sys }}$ \\
C2 & $2.39 \pm 0.08_{\text {stat }} \pm 0.12_{\text {sys }}$ \\
C2b & $2.44 \pm 0.14_{\text {stat }} \pm 0.12_{\text {sys }}$ \\
C3 & $2.35 \pm 0.06_{\text {stat }} \pm 0.11_{\text {sys }}$ \\
C4 & $2.23 \pm 0.10_{\text {stat }} \pm 0.11_{\text {sys }}$ \\
C5 & $2.27 \pm 0.11_{\text {stat }} \pm 0.11_{\text {sys }}$ \\
\hline
\end{tabular}

Extended Data Table 3: Power-law spectral indices of the $\gamma$-ray energy spectrum in different regions of the central molecular zone. The quoted errors are $1 \sigma$ statistical (stat) and systematical (sys) errors. 


\section{References}

31. Aharonian, F., et al. [H.E.S.S. Collaboration], Observations of the Crab Nebula with H.E.S.S. Astron. Astrophys. 457, 899-915 (2006).

32. de Naurois, M. \& Rolland, L., A high performance likelihood reconstruction of gamma-rays for Imaging Atmospheric Cherenkov Telescopes. Astropart. Phys. 32, 231-252 (2009).

33. Berge, D., Funk, S. \& Hinton, J. A., Background Modelling in Very-High-Energy gamma-ray Astronomy. Astron. Astrophys. 466, 1219-1229 (2007).

34. Piron, F., et al., Temporal and spectral gamma-ray properties of Mkn 421 above $250 \mathrm{GeV}$ from CAT observations between 1996 and 2000. Astron. Astrophys. 374, 895-906 (2001).

35. Ferriere, K., Gillard, W., and Jean, P., Spatial distribution of interstellar gas in the innermost 3 kpc of our Galaxy,. Astron. Astrophys. 467, 611-627 (2007)

36. Oka, T., et al., A Large-Scale CO Survey of the Galactic Center. Astrophys. J. Supp. 118, 455-515 (1998)

37. Jones, P., Burton, M., Cunningham, M., et al., Spectral imaging of the Central Molecular Zone in multiple 3-mm molecular lines. Mon. Not. Roy. Ast. Soc. 419, 2961-2986 (2012)

38. Vissani, F., Aharonian, F. \& Sahakyan, N., On the Detectability of High-Energy Galactic Neutrino Sources. Astropart. Phys. 34, 778-783 (2011).

39. Kelner, S., Aharonian, F. \& Bugayov, V., Energy spectra of gamma-rays, electrons and neutrinos produced at proton-proton interactions in the very high energy regime. Phys. Rev. D 74, 034018 (2006). [Phys. Rev. D 79, 039901 (2009)].

40. Heard, V., \& Warwick, R. S. XMM-Newton observations of the Galactic Centre Region - I. The distribution of low-luminosity X-ray sources Mon. Not. R. Ast. Soc., 428, 3462-3477 (2013)

41. Vink, J., Supernova remnants: the X-ray perspective. A\&A Rev., 20, 49 (2012).

42. Bell, A.R., Turbulent amplification of magnetic field and diffusive shock acceleration of cosmic rays. Mon. Not. R. Ast. Soc., 353, 550-558 (2004).

43. Strong, A. W., \& Moskalenko, I. V. Propagation of Cosmic-Ray Nucleons in the Galaxy. Astrophys. J., 509, 212-228 (1998).

44. Crocker, R. M., Jones, D. I., Melia, F., Ott, J., \& Protheroe, R. J. 2010. A lower limit of 50 microgauss for the magnetic field near the Galactic Centre. Nature, 463, 65-67 (2010).

45. Yusef-Zadeh, F., et al., Interacting Cosmic Rays with Molecular Clouds: A Bremsstrahlung Origin of Diffuse High Energy Emission from the Inner 2 deg by 1 deg of the Galactic Center. Astrophys. J., 762, 33 (2013). 
46. Büsching, I., de Jager, O. C. \& Snyman, J., Obtaining Cosmic-Ray Propagation Parameters from Diffuse Very High Energy Gamma-Ray Emission from the Galactic Center Ridge. Astrophys. J., 656, 841-846 (2007).

47. Yusef-Zadeh, F., et al., The Origin of Diffuse X-ray and gamma-ray Emission from the Galactic Center Region: Cosmic Ray Particles. Astrophys. J., 656, 847-869 (2007). 